\title{
TORSIONS AND INTERSECTION FORMS OF 4-MANIFOLDS FROM TRISECTION DIAGRAMS
}

\author{
VINCENT FLORENS AND DELPHINE MOUSSARD
}

\begin{abstract}
Gay and Kirby introduced trisections which describe any closed oriented smooth 4-manifold $X$ as a union of three four-dimensional handlebodies. A trisection is encoded in a diagram, namely three collections of curves in a closed oriented surface $\Sigma$, guiding the gluing of the handlebodies. Any morphism $\varphi$ from $\pi_{1}(X)$ to a finitely generated free abelian group induces a morphism on $\pi_{1}(\Sigma)$. We express the twisted homology and Reidemeister torsion of $(X ; \varphi)$ in terms of the first homology of $(\Sigma ; \varphi)$ and the three subspaces generated by the collections of curves. We also express the intersection form of $(X ; \varphi)$ in terms of the intersection form of $(\Sigma ; \varphi)$.
\end{abstract}

MSC 2010: 57Q10 57M99

\section{INTRODUCTION}

In GK16, Gay and Kirby proved that any smooth closed oriented 4-manifold can be trisected into three 4-dimensional handlebodies with 3-dimensional handlebodies as pairwise intersections and a closed surface as triple intersection. Such a decomposition can be encoded in a trisection diagram given by three families of curves on this surface. This could be thought as a 4-dimensional analogue of Heegaard splittings and diagrams and allows to use classical 2 and 3-dimensional technics to describe invariants of 4manifolds.

Feller, Klug, Schirmer and Zemke [FKSZ18] recently expressed the homology and the intersection form of a closed 4-manifold $X$ in terms of a trisection diagram. In this paper, we extend their results to the case of coefficients twisted by a group homomorphism $\varphi: \pi_{1}(X) \rightarrow G$, where $G$ is a finitely generated free abelian group, and we express the Reidemeister torsion of $(X ; \varphi)$ in terms of the diagram. More precisely, we introduce a short finite dimensional complex, whose spaces are given by the first twisted homology module of the surface $\Sigma$ of the diagram and its subspaces generated by the curves of the diagram. We show that the homology of $(X, \varphi)$ and the related Reidemeister torsion are those of this complex. Using the associated expression of the homology modules of $(X ; \varphi)$, we express the intersection form of $(X, \varphi)$ in terms of the intersection form of $(\Sigma, \varphi)$. We also give a method to compute the Alexander polynomial of $(X ; \varphi)$ from a trisection diagram.

Our approach is different from [FKSZ18]: while they use a handle decomposition of the manifold associated with the trisection, we work directly with the trisection itself - a similar method was developed by Ranicki in Ran97 to compute the signature of a 4-manifold given as a triple union. We also review the non-twisted homology and intersection form (corresponding to a trivial morphism $\varphi$ ) from this point of view; this 
yields especially a simple expression of $H_{1}(X ; \mathbb{Z})$ and explicit representatives of the homology classes.

Plan of the paper. In Section 2, we state the main results of the paper. In Section 3 , we recall some definitions and facts related to the twisted homology and the process of reconstruction of the 4-manifold $X$ from the trisection diagram; we also fix some notations. In Section 4 , we compute the homology of $X$ with coefficients in $\mathbb{Z}$. In Section 5. we describe the twisted homology of $(X ; \varphi)$ for a non-trivial $\varphi$. The torsion is treated in Sections 6 and 7 . Section 8 is devoted to intersection forms. Finally, in Section 9, we illustrate the results with explicit examples.

Acknowledgements. The first author was partially supported by the ANR Project LISA 17-CE40-0023-01. While working on the contents of this paper, the second author has been supported by a Postdoctoral Fellowship of the Japan Society for the Promotion of Science. She is grateful to Tomotada Ohtsuki and the Research Institute for Mathematical Sciences for their support. She is now supported by the Région Bourgogne Franche-Comté project ITIQ-3D. She thanks Gwénaël Massuyeau and the Institut de Mathématiques de Bourgogne for their support.

Conventions. The boundary of an oriented manifold with boundary is oriented with the "outward normal first" convention. We also use this convention to define the coorientation of an oriented manifold embedded in another oriented manifold.

We use the same notation for a curve $\nu$ in a manifold, its homotopy class and its homology class, precising the one we consider if it is not clear from the context.

If $U$ and $V$ are transverse integral chains in a manifold $M$ such that $\operatorname{dim}(U)+\operatorname{dim}(V)=$ $\operatorname{dim}(M)$, define the sign $\sigma_{x}$ of an intersection point $x \in U \cap V$ in the following way. Construct a basis of the tangent space $T_{x} M$ of $M$ at $x$ by taking an oriented basis of the normal space $N_{x} U$ followed by an oriented basis of $N_{x} V$. Set $\sigma_{x}=1$ if this basis is an oriented basis of $T_{x} M$ and $\sigma_{x}=-1$ otherwise. Now the algebraic intersection number of $U$ and $V$ in $M$ is $\langle U, V\rangle_{M}=\sum_{x \in U \cap V} \sigma_{x}$.

\section{Statement of the Results}

Let $X$ be a closed, connected, oriented, smooth 4 -manifold. A $(g, k)$-trisection of $X$ is a decomposition $X=X_{1} \cup X_{2} \cup X_{3}$ such that

- $X_{i} \simeq \natural^{k}\left(S^{1} \times B^{3}\right)$ is a 4-dimensional handlebody for each $i$,

- $X_{i} \cap X_{j} \simeq \natural^{g}\left(S^{1} \times D^{2}\right)$ is a 3 -dimensional handlebody for all $i \neq j$,

- $\Sigma=X_{1} \cap X_{2} \cap X_{3}$ is a closed surface of genus $g$.

Note that $\partial X_{i} \simeq \sharp^{k}\left(S^{1} \times S^{2}\right)$ and $\left(\Sigma, X_{i} \cap X_{i-1}, X_{i} \cap X_{i+1}\right)$ is a genus $g$ Heegaard splitting of $\partial X_{i}$, where indices are understood modulo 3. A trisection diagram consists of three systems $\left(\alpha_{i}\right)_{1 \leq i \leq g},\left(\beta_{i}\right)_{1 \leq i \leq g}$ and $\left(\gamma_{i}\right)_{1 \leq i \leq g}$ of disjoint simple closed curves on the standard closed genus $g$ surface $\Sigma$ such that each one is a complete system of meridians of a handlebody of the trisection, respectively $H_{\alpha}:=X_{3} \cap X_{1}, H_{\beta}:=X_{1} \cap X_{2}$ and $H_{\gamma}:=X_{2} \cap X_{3}$. 


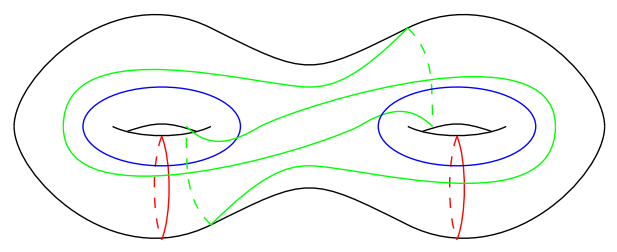

Figure 1. A trisection diagram for $S^{2} \times S^{2}$

2.1. Homology. We compute the homology of $X$ in terms of the trisection diagram. We separate the case of non-twisted coefficients (corresponding to a trivial $\varphi$ ) and the twisted case.

The first result is close to [FKSZ18, Theorem 3.1]. For $\nu \in\{\alpha, \beta, \gamma\}$, let $L_{\nu}$ be the subgroup of $H_{1}(\Sigma ; \mathbb{Z})$ generated by the homology classes of the curves $\nu_{i}$. We introduce the following complex $C$ :

$0 \rightarrow \mathbb{Z} \stackrel{0}{\longrightarrow}\left(L_{\alpha} \cap L_{\beta}\right) \oplus\left(L_{\beta} \cap L_{\gamma}\right) \oplus\left(L_{\gamma} \cap L_{\alpha}\right) \stackrel{\zeta}{\longrightarrow} L_{\alpha} \oplus L_{\beta} \oplus L_{\gamma} \stackrel{\iota}{\longrightarrow} H_{1}(\Sigma) \stackrel{0}{\longrightarrow} \mathbb{Z} \rightarrow 0$, where $\zeta(x, y, z)=(x-z, y-x, z-y)$ and $\iota$ is defined by the inclusions $L_{\nu} \hookrightarrow H_{1}(\Sigma)$ for $\nu \in\{\alpha, \beta, \gamma\}$.

Theorem 2.1. The homology of $X$ with coefficient in $\mathbb{Z}$ canonically identifies with the homology of the complex $C$. In particular:

$$
H_{1}(X) \simeq \frac{H_{1}(\Sigma)}{L_{\alpha}+L_{\beta}+L_{\gamma}} ; H_{2}(X) \simeq \frac{L_{\alpha} \cap\left(L_{\beta}+L_{\gamma}\right)}{\left(L_{\alpha} \cap L_{\beta}\right)+\left(L_{\alpha} \cap L_{\gamma}\right)} ; \quad H_{3}(X) \simeq L_{\alpha} \cap L_{\beta} \cap L_{\gamma} .
$$

The spaces in the complex $C$ can be understood as spaces of chains in $X$. This will be made explicit in Section 8 . Note that $C$ is not the same as the complex considered in [FKSZ18]: ours is symmetric in the three subspaces $L_{\alpha}, L_{\beta}$ and $L_{\gamma}$. Moreover, the expression of $H_{1}(\Sigma ; \mathbb{Z})$ was not provided in [FKSZ18.

Example 2.2. Consider the trisection diagram of $S^{2} \times S^{2}$ given in Figure 2, where the curves $\alpha$ are in red, the curves $\beta$ in blue and the curves $\gamma$ in green. One easily checks that $L_{\alpha} \cap L_{\beta} \cap L_{\gamma}=\{0\}$ and $L_{\alpha}+L_{\beta}+L_{\gamma} \simeq H_{1}(\Sigma ; \mathbb{Z})$, giving $H_{1}\left(S^{2} \times S^{2}\right)=0=H_{3}\left(S^{2} \times S^{2}\right)$. Similarly, $H_{2}\left(S^{2} \times S^{2}\right) \simeq L_{\gamma} \simeq \mathbb{Z}^{2}$.

Now fix a non-trivial morphism $\varphi: H_{1}(X ; \mathbb{Z}) \rightarrow G$, where $G$ is a finitely generated free abelian group. Thanks to Theorem 2.1, it induces a morphism $H_{1}(\Sigma ; \mathbb{Z}) \rightarrow G$, still denoted $\varphi$. Let $\mathbb{F}=\mathbb{Q}(G)$ be the quotient field of the group ring $\Lambda=\mathbb{Z}[G]$. Let $R$ stand for $\Lambda$ or $\mathbb{F}$. For $\nu \in\{\alpha, \beta, \gamma\}$, let $L_{\nu}^{R}$ be the subspace of $H_{1}^{\varphi}(\Sigma ; R)$ generated by the $R$-homology classes of the curves $\nu_{i}$. Let $C^{R}$ be the following complex:

$0 \rightarrow\left(L_{\alpha}^{R} \cap L_{\beta}^{R}\right) \oplus\left(L_{\beta}^{R} \cap L_{\gamma}^{R}\right) \oplus\left(L_{\gamma}^{R} \cap L_{\alpha}^{R}\right) \stackrel{\zeta}{\longrightarrow} L_{\alpha}^{R} \oplus L_{\beta}^{R} \oplus L_{\gamma}^{R} \stackrel{\iota}{\longrightarrow} H_{1}^{\varphi}(\Sigma ; R) \stackrel{0}{\longrightarrow} H_{0}^{\varphi}(X ; R) \rightarrow 0$, where $\zeta(x, y, z)=(x-z, y-x, z-y)$ and $\iota$ is defined by the inclusions $L_{\nu}^{R} \hookrightarrow H_{1}^{\varphi}(\Sigma ; R)$. Note that, with coefficients in $\mathbb{F}$, we have $H_{0}^{\varphi}(X ; \mathbb{F})=0$. 
Theorem 2.3. The homology of $(X ; \varphi)$ with coefficients in $R$ canonically identifies with the homology of the complex $C^{R}$. In particular, with coefficients in $R$ :

$H_{1}^{\varphi}(X) \simeq \frac{H_{1}^{\varphi}(\Sigma)}{L_{\alpha}^{R}+L_{\beta}^{R}+L_{\gamma}^{R}}, \quad H_{2}^{\varphi}(X) \simeq \frac{L_{\alpha}^{R} \cap\left(L_{\beta}^{R}+L_{\gamma}^{R}\right)}{\left(L_{\alpha}^{R} \cap L_{\beta}^{R}\right)+\left(L_{\alpha}^{R} \cap L_{\gamma}^{R}\right)}, \quad H_{3}^{\varphi}(X) \simeq L_{\alpha}^{R} \cap L_{\beta}^{R} \cap L_{\gamma}^{R}$.

This result provides in particular an expression of the Alexander module $H_{1}^{\varphi}(X ; \Lambda)$. However the $\Lambda$-module $H_{1}^{\varphi}(\Sigma ; \Lambda)$ and its submodules $L_{\nu}^{\Lambda}$ are not free modules in general, so that we do not get a free presentation of the Alexander module. However, one can compute the Alexander polynomial of $(X ; \varphi)$ using the following trick. Let $B$ be a 4 -ball in $X$ that intersects $\Sigma$ transversely along a disk $D$ disjoint from the $3 g$ curves of the diagram. Set $\widehat{X}=X \backslash \operatorname{Int}(B)$ and $\widehat{\Sigma}=\Sigma \backslash \operatorname{Int}(D)$. Fix a base-point $\star \in \partial D$. One easily checks that the $\Lambda$-modules $H_{1}^{\varphi}(X ; \Lambda)$ and $H_{1}^{\varphi}(\widehat{X}, \star ; \Lambda)$ have the same $\Lambda$-torsion submodule, so that $(X ; \varphi)$ and $(\widehat{X}, \star ; \varphi)$ have the same Alexander polynomial.

For $\nu \in\{\alpha, \beta, \gamma\}$, let $\widehat{L}_{\nu}^{\Lambda}$ be the subspace of $H_{1}^{\varphi}(\widehat{\Sigma}, \star ; \Lambda)$ generated by the homology classes of the curves $\nu_{i}$. We show in Lemma 7.1 that $H_{1}^{\varphi}(\widehat{\Sigma}, \star ; \Lambda), \widehat{L}_{\nu}^{\Lambda}$ and $\widehat{L}_{\nu}^{\Lambda} \cap \widehat{L}_{\nu^{\prime}}^{\Lambda}$ are free $\Lambda$-modules. As previously, we consider a complex of $\Lambda$-modules $\widehat{C}^{\Lambda}$ :

$$
0 \rightarrow\left(\widehat{L}_{\alpha}^{\Lambda} \cap \widehat{L}_{\beta}^{\Lambda}\right) \oplus\left(\widehat{L}_{\beta}^{\Lambda} \cap \widehat{L}_{\gamma}^{\Lambda}\right) \oplus\left(\widehat{L}_{\gamma}^{\Lambda} \cap \widehat{L}_{\alpha}^{\Lambda}\right) \stackrel{\zeta}{\longrightarrow} \widehat{L}_{\alpha}^{\Lambda} \oplus \widehat{L}_{\beta}^{\Lambda} \oplus \widehat{L}_{\gamma}^{\Lambda} \stackrel{\iota}{\longrightarrow} H_{1}^{\varphi}(\widehat{\Sigma}, \star ; \Lambda) \rightarrow 0 .
$$

With the very same proof as for Theorem 2.3, one shows the following result.

Theorem 2.4. The $\Lambda$-homology of $(\widehat{X}, \star ; \varphi)$ canonically identifies with the homology of the complex $\widehat{C}^{\Lambda}$. In particular, the Alexander $\Lambda$-module of $(\widehat{X}, \star ; \varphi)$ admits the finite presentation:

$$
\widehat{L}_{\alpha}^{\Lambda} \oplus \widehat{L}_{\beta}^{\Lambda} \oplus \widehat{L}_{\gamma}^{\Lambda} \rightarrow H_{1}^{\varphi}(\widehat{\Sigma}, \star ; \Lambda) \rightarrow H_{1}^{\varphi}(\widehat{X}, \star ; \Lambda) \rightarrow 0 .
$$

This result provides a presentation matrix of the Alexander $\Lambda$-module of $(\widehat{X}, \star ; \varphi)$ from which one can compute the Alexander polynomial of $(\widehat{X}, \star ; \varphi)$ and $(X ; \varphi)$.

2.2. Intersection forms. For $R=\Lambda$ or $\mathbb{F}$, we express the intersection form of $(X ; \varphi)$ using the expression of $H_{2}^{\varphi}(X ; R)$ given by Theorem 2.3 and the intersection form of $(\Sigma ; \varphi)$. The spaces $L_{\nu}^{R}$ coincide with

$$
\operatorname{ker}\left(H_{1}^{\varphi}(\Sigma ; R) \stackrel{i n c l_{*}}{\longrightarrow} H_{1}^{\varphi}\left(H_{\nu} ; R\right)\right) .
$$

Define the hermitian form

$$
\lambda^{\varphi}: \frac{L_{\alpha}^{R} \cap\left(L_{\beta}^{R}+L_{\gamma}^{R}\right)}{\left(L_{\alpha}^{R} \cap L_{\beta}^{R}\right)+\left(L_{\alpha}^{R} \cap L_{\gamma}^{R}\right)} \times \frac{L_{\alpha}^{R} \cap\left(L_{\beta}^{R}+L_{\gamma}^{R}\right)}{\left(L_{\alpha}^{R} \cap L_{\beta}^{R}\right)+\left(L_{\alpha}^{R} \cap L_{\gamma}^{R}\right)} \longrightarrow R
$$

as follows. For $a, a^{\prime} \in L_{\alpha}^{R} \cap\left(L_{\beta}^{R}+L_{\gamma}^{R}\right)$ and $b \in L_{\beta}^{R}, c \in L_{\gamma}^{R}$ such that $a+b+c=0$, set

$$
\lambda^{\varphi}\left(a, a^{\prime}\right):=\left\langle c, a^{\prime}\right\rangle_{\Sigma}^{\varphi}
$$

Note that permuting the roles of $\alpha, \beta$ and $\gamma$ in this construction gives the same form, up to the sign of the permutation. This is related to the fact that the coorientation of $\Sigma$ - defined by the orientations of $\Sigma$ and $X$ - induces a cyclic order on the $X_{i}$ and the $H_{\nu}$. 
Theorem 2.5. Let $\langle\cdot, \cdot\rangle_{X}^{\varphi}$ be the intersection form of $(X ; \varphi)$. There is an isomorphism

$$
\left(H_{2}^{\varphi}(X ; R),\langle\cdot, \cdot\rangle_{X}^{\varphi}\right) \simeq\left(\frac{L_{\alpha}^{R} \cap\left(L_{\beta}^{R}+L_{\gamma}^{R}\right)}{\left(L_{\alpha}^{R} \cap L_{\beta}^{R}\right)+\left(L_{\alpha}^{R} \cap L_{\gamma}^{R}\right)}, \lambda^{\varphi}\right) .
$$

The form $\lambda^{\varphi}$ is a hermitian version of a symmetric form introduced by Wall in [Wal69], which is involved in the similar result in the non-twisted setting [FKSZ18, Theorem 3.6], corresponding to a trivial morphism $\varphi$. As noted in [FKSZ18, Remark 3.7], the main theorem of Wal69 implies that the signature of the intersection form of $(X ; \varphi)$ equals the signature of the form $\lambda^{\varphi}$. In the case of trisections, the above theorem says that not only the signatures coincide, but also the forms themselves.

Proposition 2.6. There is an isomorphism

$$
\left(H_{1}^{\varphi}(X ; R) \times H_{3}^{\varphi}(X ; R) ;\langle\cdot, \cdot\rangle_{X}^{\varphi}\right) \simeq\left(\frac{H_{1}^{\varphi}(\Sigma)}{L_{\alpha}^{R}+L_{\beta}^{R}+L_{\gamma}^{R}} \times\left(L_{\alpha}^{R} \cap L_{\beta}^{R} \cap L_{\gamma}^{R}\right) ;\langle\cdot, \cdot\rangle_{\Sigma}^{\varphi}\right) .
$$

2.3. Abelian torsions. We now state the result for the torsion. Consider the complex $C^{\mathbb{F}}$ defined before Theorem 2.3 ,

Theorem 2.7. There exists an $\mathbb{F}$-basis $c$ for $C^{\mathbb{F}}$ such that for any homology $\mathbb{F}$-basis $h$ of $X$ and $C^{\mathbb{F}}$, the following holds:

$$
\tau^{\varphi}(X ; h)=\tau\left(C^{\mathbb{F}} ; c, h\right) \quad \text { in } \mathbb{F} / \pm \varphi\left(H_{1}(X)\right) .
$$

The complex basis $c$ is explicited in Subsection 6.1. Although the bases for $H_{1}^{\varphi}(\Sigma ; \mathbb{F})$ and the $L_{\nu}^{\mathrm{F}}$ are straightforwardly obtained from the trisection diagram, the computation of the bases for the intersections $L_{\nu}^{\mathbb{F}} \cap L_{\nu^{\prime}}^{\mathbb{F}}$ involves handleslides on the surface. From an algorithmic point of view, this might not be efficient. As an alternative way, one may use the same trick as in Section 2.1 and compute the torsion of $(\widehat{X}, \star)$ instead, where $\widehat{X}$ is the complement of 4-ball and $\star$ is a base point in the boundary. The two torsions $\tau^{\varphi}(X)$ and $\tau^{\varphi}(\widehat{X}, \star)$ coincide up to a factor, see Proposition 7.4. This allows to use much more general complex bases, avoiding the handleslides, see Theorem 7.2 . The (light) price to pay is that $\tau^{\varphi}(\widehat{X}, \star)$ is computed only up to a unit in $\Lambda$.

\section{Preliminaries}

3.1. Algebraic torsion. We recall the algebraic setup, see [Mil66] and Tur01 for further details and references. Let $\mathbb{K}$ be a field. If $V$ is a finite dimensional $\mathbb{K}$-vector space and $b$ and $c$ are two bases of $V$, we denote by $[b / c]$ the determinant of the matrix expressing the basis change from $b$ to $c$. The bases $b$ and $c$ are equivalent if $[b / c]=1$. Let $C$ be a finite complex of finite dimensional $\mathbb{K}$-vector spaces:

$$
C=\left(C_{m} \stackrel{\partial_{m}}{\longrightarrow} C_{m-1} \longrightarrow \cdots \stackrel{\partial_{1}}{\longrightarrow} C_{0}\right) .
$$

A complex basis of $C$ is a family $c=\left(c_{m}, \ldots, c_{0}\right)$ where $c_{i}$ is a basis of $C_{i}$ for all $i \in\{0, \ldots, m\}$. A homology basis of $C$ is a family $h=\left(h_{m}, \ldots, h_{0}\right)$ where $h_{i}$ is a basis of the homology group $H_{i}(C)$ for all $i \in\{0, \ldots, m\}$. If we have chosen a basis $b_{j}$ of the space of $j$-dimensional boundaries $B_{j}(C):=\operatorname{Im} \partial_{j+1}$ for all $j \in\{0, \ldots, m-1\}$, then a homology basis $h$ of $C$ induces an equivalence class of bases $\left(b_{i} h_{i}\right) b_{i-1}$ of $C_{i}$ for all $i$. 
The torsion of the $\mathbb{K}$-complex $C$, equipped with a complex basis $c$ and a homology basis $h$, is the scalar:

$$
\tau(C ; c, h):=\prod_{i=0}^{m}\left[\left(b_{i} h_{i}\right) b_{i-1} / c_{i}\right]^{(-1)^{i+1}} \in \mathbb{K}^{*} .
$$

It is easily checked that this definition does not depend on the choice of $b_{0}, \ldots, b_{m}$. When $C$ is acyclic, we set $\tau(C ; c):=\tau(C ; c, \varnothing)$.

Lemma 3.1. Consider a short exact sequence $0 \rightarrow C^{\prime} \rightarrow C \rightarrow C^{\prime \prime} \rightarrow 0$ of $\mathbb{K}$-complexes with compatible complex bases $c^{\prime}, c, c^{\prime \prime}$ in the sense that $c_{i}$ is equivalent to $c_{i}^{\prime} c_{i}^{\prime \prime}$ for every $i \in$ $\{0, \ldots, m\}$ and homology bases $h^{\prime}, h, h^{\prime \prime}$. The associated long exact sequence in homology $\mathcal{H}$ is an acyclic finite $\mathbb{K}$-complex with base $\left(h^{\prime}, h, h^{\prime \prime}\right):=\left(h_{m}^{\prime}, h_{m}, h_{m}^{\prime \prime}, \ldots, h_{0}^{\prime}, h_{0}, h_{0}^{\prime \prime}\right)$ and we have

$$
\tau(C ; c, h)=\varepsilon \cdot \tau\left(C^{\prime} ; c^{\prime}, h^{\prime}\right) \cdot \tau\left(C^{\prime \prime} ; c^{\prime \prime}, h^{\prime \prime}\right) \cdot \tau\left(\mathcal{H} ;\left(h^{\prime}, h, h^{\prime \prime}\right)\right),
$$

where $\varepsilon$ is a sign depending on the dimensions of $C_{i}^{\prime}, C_{i}, C_{i}^{\prime \prime}$ and $H_{i}\left(C^{\prime}\right), H_{i}(C), H_{i}\left(C^{\prime \prime}\right)$ for $i \in\{0, \ldots, m\}$.

3.2. Twisted homology and Reidemeister torsion. Let $(X, Y)$ be a finite CW-pair with maximal abelian cover $p: \bar{X} \rightarrow X$. Let $G$ be a finitely generated free abelian group. Fix a group homomorphism $\varphi: \pi_{1}(X) \rightarrow G$ and denote $R$ the group ring $\Lambda=\mathbb{Z}[G]$ or its quotient field $\mathbb{F}=\mathbb{Q}(G)$. The extension of $\varphi$ to a ring morphism $\mathbb{Z}\left[H_{1}(X)\right] \rightarrow R$ is still denoted $\varphi$. The chain complex of $(X, Y ; \varphi)$ with coefficient in $R$ is defined as

$$
C^{\varphi}(X, Y ; R)=C\left(\bar{X}, p^{-1}(Y)\right) \otimes_{\mathbb{Z}\left[H_{1}(X)\right]} R .
$$

We denote $H^{\varphi}(X, Y ; R)$ its homology. It is easy to check that

$$
H^{\varphi}(X, Y ; \mathbb{F})=H^{\varphi}(X, Y ; \Lambda) \otimes_{\Lambda} \mathbb{F} .
$$

Let $\bar{c}$ be a complex basis of the complex of free $\mathbb{Z}\left[H_{1}(X)\right]$-module $C\left(\bar{X}, p^{-1}(Y)\right)$ obtained by lifting each relative cell of $(X, Y)$ to $\bar{X}$. Then $c=\bar{c} \otimes 1$ is a complex basis of $C^{\varphi}(X, Y ; \mathbb{F})$.

Definition 3.2. Given a homology basis $h$ of $H^{\varphi}(X, Y ; \mathbb{F})$, the torsion of $(X, Y ; \varphi)$ is

$$
\tau^{\varphi}(X, Y ; h):=\tau\left(C^{\varphi}(X, Y ; \mathbb{F}) ; c, h\right) \in \mathbb{F} / \pm \varphi\left(H_{1}(X)\right) .
$$

The ambiguity in $\pm \varphi\left(H_{1}(X)\right)$ is due to the different choices of lift and orientation of the cells. Note that the torsion of $(X, Y ; \varphi)$ is closely related to the orders of the $\Lambda$-modules $H^{\varphi}(X, Y ; \Lambda)$, see [KL99].

We end the subsection with two useful results.

Lemma 3.3. If $X$ is connected and $\varphi$ is non-trivial, then $H_{0}^{\varphi}(X ; \mathbb{F})=0$.

By Blanchfield duality (see Subsection 3.3), Lemma 3.3 implies the following corollary.

Corollary 3.4. Assume $X$ is a compact connected oriented $n$-manifold. If $\varphi$ is nontrivial, then $H_{n}^{\varphi}(X, \partial X ; \mathbb{F})=0$. 


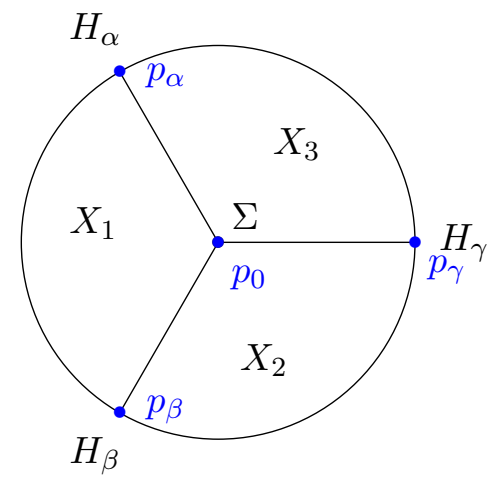

Figure 2. Decomposition of $X$.

3.3. Twisted intersection form and Blanchfield duality. Let $W$ be a compact oriented $n$-manifold and $\varphi: \pi_{1}(W) \rightarrow G$ be a group homomorphism. For $q \in\{0, \ldots, n\}$, the twisted intersection form of $W$ with coefficient in $R$, introduced by Reidemeister in Rei39, is the sesquilinear map

$$
\langle\cdot, \cdot\rangle_{W}^{\varphi}: H_{q}^{\varphi}(W ; R) \times H_{n-q}^{\varphi}(W, \partial W ; R) \longrightarrow R
$$

defined by

$$
\left\langle x \otimes z, x^{\prime} \otimes z^{\prime}\right\rangle_{W}^{\varphi}=\sum_{h \in \frac{H_{1}(W)}{\operatorname{ker}(\varphi)}}\left\langle x, h \cdot x^{\prime}\right\rangle_{W} \varphi(h) \otimes z z^{\prime},
$$

where $R=\Lambda$ or $\mathbb{F}, \bar{W} \rightarrow W$ is the covering associated with $\operatorname{ker}(\varphi)$ and $\langle\cdot, \cdot\rangle_{\bar{W}}$ stands for the algebraic intersection in $\bar{W}$. By Blanchfield's duality theorem [Bla57, Theorem 2.6], for $R=\mathbb{F}$, this form is nondegenerate.

3.4. Reconstruction of $X$ from the trisection diagram. A trisection diagram determines the associated 4-manifold. We recall here how to reconstruct the manifold from the diagram and we introduce some notations that we will use in the next sections.

We are given a trisection diagram, i.e. a closed genus $g$ surface $\Sigma$ and three systems of meridians $\left(\alpha_{i}\right)_{1 \leq i \leq g},\left(\beta_{i}\right)_{1 \leq i \leq g}$, and $\left(\gamma_{i}\right)_{1 \leq i \leq g}$. Consider a disk $D^{2}$ with center $p_{0}$ and three distinct points $p_{\alpha}, p_{\beta}, p_{\gamma}$ on the boundary, see Figure 2, Take the product $D^{2} \times \Sigma$ and add a $2-$ cell along $p_{\nu} \times \nu_{i}$ for all $\nu=\alpha, \beta, \gamma$ and all $1 \leq i \leq g$. It remains to add 3-cells and 4-cells; the way this is performed as no incidence, see Laudenbach and Poénaru [LP72]. In this decomposition of $X, H_{\nu}$ is recovered as $\left[p_{0}, p_{\nu}\right] \times \Sigma$ union the corresponding 2-cells and 3-cell. The union $Y=H_{\alpha} \cup H_{\beta} \cup H_{\gamma}$ is the spine of the trisection. In the computations of homology and torsion, we will make a great use of the exact sequences in homology associated with the pairs $(Y, \Sigma)$ and $(X, Y)$.

\section{Homology of $X$}

Throughout this section, $H_{\ell}($.$) stands for the homology with coefficients in \mathbb{Z}$. We fix a trisection $X=X_{1} \cup X_{2} \cup X_{3}$ given by a diagram $\left(\Sigma ; \alpha, \beta\right.$, $\gamma$ ), with spine $Y=H_{\alpha} \cup H_{\beta} \cup H_{\gamma}$ (see Section 3.4). We prove Theorem 2.1 using the pairs $(Y, \Sigma)$ and $(X, Y)$. 
Lemma 4.1.

$$
H_{\ell}(Y, \Sigma) \simeq\left\{\begin{array}{cc}
L_{\alpha} \oplus L_{\beta} \oplus L_{\gamma} & \text { if } \ell=2 \\
\mathbb{Z}^{3} & \text { if } \ell=3 \\
0 & \text { otherwise }
\end{array}\right.
$$

Proof. Note that $H_{\ell}(Y, \Sigma) \simeq H_{\ell}\left(H_{\alpha}, \Sigma\right) \oplus H_{\ell}\left(H_{\beta}, \Sigma\right) \oplus H_{\ell}\left(H_{\gamma}, \Sigma\right)$ and:

$$
H_{\ell}\left(H_{\nu}, \Sigma\right) \simeq\left\{\begin{array}{cc}
L_{\nu} & \text { if } \ell=2 \\
\mathbb{Z} & \text { if } \ell=3 \\
0 & \text { otherwise }
\end{array}\right.
$$

thanks to the exact sequence associated with $\left(H_{\nu}, \Sigma\right)$.

Lemma 4.2. The homology of $Y$ with coefficients in $\mathbb{Z}$ is given by

$$
H_{1}(Y) \simeq \frac{H_{1}(\Sigma)}{L_{\alpha}+L_{\beta}+L_{\gamma}} ; H_{2}(Y) \simeq \operatorname{ker}\left(L_{\alpha} \oplus L_{\beta} \oplus L_{\gamma} \rightarrow H_{1}(\Sigma)\right) ; H_{3}(Y) \simeq \mathbb{Z}^{2} .
$$

Proof. We use the exact sequence associated with the pair $(Y, \Sigma)$. Since the surface $\Sigma$ bounds any of the $H_{\nu}$ in $Y$, the map $H_{2}(\Sigma) \rightarrow H_{2}(Y)$ is trivial and we get:

$$
0 \rightarrow H_{3}(Y) \rightarrow H_{3}(Y, \Sigma) \rightarrow H_{2}(\Sigma) \rightarrow 0,
$$

from which we easily see that $H_{3}(Y) \simeq \mathbb{Z}^{2}$, generated by two of the boundaries $\partial X_{i}$, and:

$$
0 \rightarrow H_{2}(Y) \rightarrow H_{2}(Y, \Sigma) \rightarrow H_{1}(\Sigma) \rightarrow H_{1}(Y) \rightarrow 0
$$

which provides the expressions of $H_{1}(Y)$ and $H_{2}(Y)$, using Lemma 4.1.

\section{Lemma 4.3.}

$$
H_{\ell}(X, Y) \simeq\left\{\begin{array}{cc}
\left(L_{\alpha} \cap L_{\beta}\right) \oplus\left(L_{\beta} \cap L_{\gamma}\right) \oplus\left(L_{\gamma} \cap L_{\alpha}\right) & \text { if } \ell=3 \\
\mathbb{Z}^{3} & \text { if } \ell=4 \\
0 & \text { otherwise }
\end{array}\right.
$$

Proof. Note that $H_{\ell}(X, Y) \simeq \oplus_{j=1}^{3} H_{\ell}\left(X_{j}, \partial X_{j}\right)$. One easily checks that $H_{4}\left(X_{j}, \partial X_{j}\right) \simeq$ $\mathbb{Z}$ and $H_{\ell}\left(X_{j}, \partial X_{j}\right)=0$ if $\ell \neq 3,4$. For $\ell=3$, the exact sequence associated with $\left(X_{j}, \partial X_{j}\right)$ gives $H_{3}\left(X_{j}, \partial X_{j}\right) \simeq H_{2}\left(\partial X_{j}\right)$. Now, for $\nu, \nu^{\prime} \in\{\alpha, \beta, \gamma\}$ such that $\partial X_{j}=$ $H_{\nu} \cup_{\Sigma} H_{\nu^{\prime}}$, the Mayer-Vietoris sequence gives:

$$
0 \rightarrow H_{2}\left(\partial X_{j}\right) \rightarrow H_{1}(\Sigma) \rightarrow H_{1}\left(H_{\nu}\right) \oplus H_{1}\left(H_{\nu^{\prime}}\right) .
$$

Hence $H_{2}\left(\partial X_{j}\right) \simeq L_{\nu} \cap L_{\nu^{\prime}}$.

Proof of Theorem 2.1. We use the exact sequence associated with the pair $(X, Y)$. The map $H_{3}(Y) \rightarrow H_{3}(X)$ is trivial since $H_{3}(Y)$ is generated by the classes of the $\partial X_{j}$ for $j \in\{1,2,3\}$, which bound the $X_{j}$ in $X$. Thus

$$
0 \rightarrow H_{3}(X) \rightarrow H_{3}(X, Y) \stackrel{\zeta}{\longrightarrow} H_{2}(Y) \rightarrow H_{2}(X) \rightarrow 0
$$

and $H_{1}(Y) \simeq H_{1}(X)$, thanks to Lemma 4.3. The expression of the map $\zeta$ follows of the descriptions of $H_{2}(Y)$ in Lemma 4.2 and of $H_{3}(X, Y)$ in Lemma 4.3 .

We end this section with a lemma which will be useful in the next sections. 
Lemma 4.4. If $G$ is a finitely generated abelian group and $\varphi: H_{1}(X) \rightarrow G$ a non-trivial morphism, then $\varphi$ induces non-trivial morphisms on the first homology groups of the spaces $\Sigma, H_{\nu}$ for $\nu \in\{\alpha, \beta, \gamma\}, Y, X_{i}$ and $\partial X_{i}$ for $i=1,2,3$.

Proof. For $Y$, it is obvious since $H_{1}(Y) \simeq H_{1}(X)$. Thanks to the expression of $H_{1}(X)$ given in Theorem 2.1, the homology groups $H_{1}(\Sigma), H_{1}\left(H_{\nu}\right) \simeq H_{1}(\Sigma) / L_{\nu}$ and $H_{1}\left(X_{1}\right) \simeq$ $H_{1}\left(\partial X_{1}\right) \simeq H_{1}(\Sigma) /\left(L_{\alpha} \cap L_{\beta}\right)$ naturally surject on $H_{1}(X)$. Compose $\varphi$ by these surjections.

For simplicity all these morphisms induced by $\varphi$ are still denoted $\varphi$.

\section{Homology of $(X ; \varphi)$}

In this section, we compute the homology of $(X ; \varphi)$ for a non-trivial $\varphi$. As in Section 4 , we use the pairs $(Y, \Sigma)$ and $(X, Y)$. The notation $R$ is used for either $\Lambda$ or $\mathbb{F}$ and $H^{\varphi}(\cdot)$ stands for the homology with coefficients in $R$.

Lemma 5.1. For any $\nu \in\{\alpha, \beta, \gamma\}, L_{\nu}^{R}=\operatorname{ker}\left(H_{1}^{\varphi}(\Sigma) \stackrel{i n c l_{*}}{\longrightarrow} H_{1}^{\varphi}\left(H_{\nu}\right)\right)$.

Proof. Let $\nu \in\{\alpha, \beta, \gamma\}$. Since $H_{\nu}$ retracts on a wedge of circles, we have $H_{2}^{\varphi}\left(H_{\nu}\right)=0$ and the exact sequence in homology of the pair $\left(H_{\nu}, \Sigma\right)$ provides the short exact sequence:

$$
0 \rightarrow H_{2}^{\varphi}\left(H_{\nu}, \Sigma\right) \rightarrow H_{1}^{\varphi}(\Sigma) \rightarrow H_{1}^{\varphi}\left(H_{\nu}\right)
$$

Now, $H_{\nu}$ is obtained from $\Sigma$ by adding meridian disks $D_{i}^{\nu}$ such that $\partial D_{i}^{\nu}=\nu_{i}$ and one 3-cell. Hence $H_{2}^{\varphi}\left(H_{\nu}, \Sigma\right)$ is generated by the $D_{i}^{\nu}$ for $i=1, \ldots, g$ and its image in $H_{1}^{\varphi}(\Sigma)$ is exactly the submodule $L_{\nu}^{R}$ generated by the $\nu_{i}$.

Lemma 5.2. We have $H_{\ell}^{\varphi}(Y, \Sigma)=0$ for $\ell \neq 2$. Moreover, there is a natural identification

$$
H_{2}^{\varphi}(Y, \Sigma) \simeq L_{\alpha}^{R} \oplus L_{\beta}^{R} \oplus L_{\gamma}^{R}
$$

Proof. Use $H_{\ell}^{\varphi}(Y, \Sigma) \simeq H_{\ell}^{\varphi}\left(H_{\alpha}, \Sigma\right) \oplus H_{\ell}^{\varphi}\left(H_{\beta}, \Sigma\right) \oplus H_{\ell}^{\varphi}\left(H_{\gamma}, \Sigma\right)$ and the exact sequence associated with $\left(H_{\nu}, \Sigma\right)$.

Lemma 5.3. There are natural identifications:

$$
\begin{aligned}
& H_{1}^{\varphi}(Y) \simeq \frac{H_{1}^{\varphi}(\Sigma)}{L_{\alpha}^{R}+L_{\beta}^{R}+L_{\gamma}^{R}}, \\
& H_{2}^{\varphi}(Y) \simeq \operatorname{ker}\left(L_{\alpha}^{R} \oplus L_{\beta}^{R} \oplus L_{\gamma}^{R} \rightarrow H_{1}^{\varphi}(\Sigma)\right) .
\end{aligned}
$$

Proof. Thanks to Lemma 5.2, the exact sequence in homology of the pair $(Y, \Sigma)$ reduces to

$$
0 \rightarrow H_{2}^{\varphi}(Y) \longrightarrow L_{\alpha}^{R} \oplus L_{\beta}^{R} \oplus L_{\gamma}^{R} \rightarrow H_{1}^{\varphi}(\Sigma) \longrightarrow H_{1}^{\varphi}(Y) \longrightarrow 0 .
$$

This provides the given expressions for the homology of $Y$.

Lemma 5.4. We have $H_{\ell}^{\varphi}(X, Y)=0$ for $\ell \neq 3$. Moreover, there is a natural identification

$$
H_{3}^{\varphi}(Y, \Sigma) \simeq\left(L_{\alpha}^{R} \cap L_{\beta}^{R}\right) \oplus\left(L_{\beta}^{R} \cap L_{\gamma}^{R}\right) \oplus\left(L_{\gamma}^{R} \cap L_{\alpha}^{R}\right) .
$$


Proof. Note that $H_{\ell}^{\varphi}(X, Y) \simeq \oplus_{j=1}^{3} H_{\ell}^{\varphi}\left(X_{j}, \partial X_{j}\right)$. Let's focuse on $\left(X_{1}, \partial X_{1}\right)$. For $\ell=3$, the exact sequence associated with $\left(X_{1}, \partial X_{1}\right)$ gives $H_{3}\left(X_{1}, \partial X_{1}\right) \simeq H_{2}\left(\partial X_{1}\right)$. Now, the Mayer-Vietoris sequence associated with the Heegaard splitting $\partial X_{1}=H_{\alpha} \cup_{\Sigma} H_{\beta}$ gives:

$$
0 \rightarrow H_{2}^{\varphi}\left(\partial X_{1}\right) \rightarrow H_{1}^{\varphi}(\Sigma) \stackrel{f}{\longrightarrow} H_{1}^{\varphi}\left(H_{\alpha}\right) \oplus H_{1}^{\varphi}\left(H_{\beta}\right) .
$$

We get $H_{2}^{\varphi}\left(\partial X_{1}\right) \simeq \operatorname{ker}(f)=L_{\alpha}^{R} \cap L_{\beta}^{R}$.

Proof of Theorem 2.3. The exact sequence associated with the pair $(X, Y)$ gives

$$
0 \rightarrow H_{3}^{\varphi}(X) \rightarrow H_{3}^{\varphi}(X, Y) \stackrel{\zeta}{\longrightarrow} H_{2}^{\varphi}(Y) \rightarrow H_{2}^{\varphi}(X) \rightarrow 0
$$

and $H_{1}^{\varphi}(X) \simeq H_{1}^{\varphi}(Y)$. The result then follows from Lemmas 5.3 and 5.4

\section{TORsion of $(X ; \varphi)$}

In this section, $H^{\varphi}(\cdot)$ stands for the twisted homology with coefficients in $\mathbb{F}$ and we assume that $\varphi$ is non-trivial. The aim of the section is to prove Theorem 2.7. We fix a lift of a given 0 -cell $\star$ of $X$ and require that the lift of any 1 -cell starts at the chosen lift of $\star$.

6.1. Bases in homology and first computations. In this subsection, we define bases for the complex $C^{\mathbb{F}}$ of Theorem 2.7 and we compute some related torsions that we need to prove the theorem.

Definition 6.1. A (geometric) symplectic basis of $\Sigma$ is a family $\left(x_{i}, y_{i}\right)_{1 \leq i \leq g}$ of simple closed curves in $\Sigma$, based at $\star$, such that

- any two curves meet only at $\star$,

- the classes of $x_{i}$ and $y_{i}$ form a basis of $H_{1}(\Sigma ; \mathbb{Z})$, symplectic with respect to the intersection form $\langle., .\rangle_{\Sigma}$,

- there exists a CW-complex decomposition of $\Sigma$ with a single 2-cell glued along

$$
\partial \Sigma=\prod_{i=1}^{g}\left[x_{i}, y_{i}\right] .
$$

Lemma 6.2. Let $\left(x_{i}, y_{i}\right)_{1 \leq i \leq g}$ be a symplectic basis of $\Sigma$ such that $x_{i} \in \operatorname{ker}(\varphi)$ for all $i$. $U p$ to re-ordering, assume that $y_{1} \notin \operatorname{ker}(\varphi)$. For $i=2, \ldots, g$, set

$$
y_{i}^{\prime}=y_{i}-\frac{\varphi\left(y_{i}\right)-1}{\varphi\left(y_{1}\right)-1} y_{1} \in C_{1}^{\varphi}(\Sigma) .
$$

Then the family $h_{\Sigma}=\left(x_{i}, y_{i}^{\prime}\right)_{i>1}$ is a basis of $H_{1}^{\varphi}(\Sigma)$ and $\tau^{\varphi}\left(\Sigma ; h_{\Sigma}\right)=1$.

For instance, in Lemma 6.2, one can choose the family $\left(x_{i}\right)_{i}$ to coincide with the family $\alpha, \beta$ or $\gamma$ and $\left(y_{i}\right)_{i}=\left(x_{i}^{*}\right)_{i}$ to be a dual family. Note that $H_{1}^{\varphi}(\Sigma)=0$ if $g=1$.

Proof. There is a CW-decomposition of $\Sigma$ given by $\star$ as 0 -cell, the $x_{i}$ and $y_{i}$ as 1-cell and $\Sigma$ as 2-cell. The associated $\mathbb{F}$-complex is $C^{\varphi}(\Sigma): 0 \rightarrow C_{2}^{\varphi}(\Sigma) \rightarrow C_{1}^{\varphi}(\Sigma) \rightarrow C_{0}^{\varphi}(\Sigma) \rightarrow 0$ with basis $\Sigma,\left(x_{i}, y_{i}\right)_{i}$ and $\star$. We choose the lift of $\Sigma$ so that

$$
\partial \Sigma=\sum_{1 \leq i \leq g}\left(\varphi\left(y_{i}\right)-1\right) x_{i} \in C_{1}^{\varphi}(\Sigma) .
$$


TORSIONS AND INTERSECTION FORMS OF 4-MANIFOLDS FROM TRISECTION DIAGRAMS 11

For all $i, \partial x_{i}=0$ and $\partial y_{i}=\left(\varphi\left(y_{i}\right)-1\right) \star$. Hence $h_{\Sigma}$ is a basis of $H_{1}^{\varphi}(\Sigma)$. We get

$$
\tau^{\varphi}\left(\Sigma ; h_{\Sigma}\right)=\left[\frac{\partial \Sigma \cdot h_{\Sigma} \cdot\left(\varphi\left(y_{1}\right)-1\right)^{-1} y_{1}}{\left(x_{i}, y_{i}\right)_{1 \leq i \leq g}}\right]=1 \text {. }
$$

Lemma 6.3. Let $\nu \in\{\alpha, \beta, \gamma\}$ and $\left(\nu_{i}^{*}\right)_{1 \leq i \leq g}$ be simple closed curves in $\Sigma$ such that $\left(\nu_{i}, \nu_{i}^{*}\right)_{1 \leq i \leq g}$ is a symplectic basis for $\Sigma$. Permuting the indices if necessary, assume that $\varphi\left(\nu_{1}^{*}\right) \neq 1$. Then the family $h_{\nu}=\left(\frac{1}{\varphi\left(\nu_{1}^{*}\right)-1} \nu_{2}, \nu_{3}, \ldots, \nu_{g}\right)$ is a basis of $L_{\nu}^{\mathbb{F}}$.

Proof. By definition, $L_{\nu}^{\mathbb{F}}$ is generated by the $\nu_{i}$. Consider the same complex as in the proof of Lemma 6.2, with $x_{i}=\nu_{i}$ and $y_{i}=\nu_{i}^{*}$. The only relation is $\sum_{1 \leq i \leq g}\left(\varphi\left(\nu_{i}^{*}\right)-1\right) \nu_{i}=0$.

Lemma 6.4. Via the identification $H_{2}^{\varphi}(Y, \Sigma) \simeq L_{\alpha}^{\mathbb{F}} \oplus L_{\beta}^{\mathbb{F}} \oplus L_{\gamma}^{\mathbb{F}}$, the family $h_{Y, \Sigma}=h_{\alpha} \cdot h_{\beta} \cdot h_{\gamma}$ is a homology basis of $(Y, \Sigma ; \varphi)$ and we have $\tau^{\varphi}\left(Y, \Sigma ; h_{Y, \Sigma}\right)=1$.

Proof. By Lemma 5.2, $H_{2}^{\varphi}(Y, \Sigma) \simeq L_{\alpha}^{\mathbb{F}} \oplus L_{\beta}^{\mathbb{F}} \oplus L_{\gamma}^{\mathbb{F}}$ is the only non-trivial space in $H^{\varphi}(Y, \Sigma)$. Moreover, the isomorphism of chain complexes $C^{\varphi}(Y, \Sigma) \simeq C^{\varphi}\left(H_{\alpha}, \Sigma\right) \oplus C^{\varphi}\left(H_{\beta}, \Sigma\right) \oplus$ $C^{\varphi}\left(H_{\gamma}, \Sigma\right)$ provides

$$
\tau^{\varphi}\left(Y, \Sigma ; h_{Y, \Sigma}\right)=\tau^{\varphi}\left(H_{\alpha}, \Sigma ; h_{\alpha}\right) \tau^{\varphi}\left(H_{\beta}, \Sigma ; h_{\beta}\right) \tau^{\varphi}\left(H_{\gamma}, \Sigma ; h_{\gamma}\right) .
$$

Fix $\nu \in\{\alpha, \beta, \gamma\}$. The handlebody $H_{\nu}$ is obtained from $\Sigma$ by adding meridian disks $D_{i}^{\nu}$ such that $\partial D_{i}^{\nu}=\nu_{i}$ and the 3 -cell $H_{\nu}$. The associated $\mathbb{F}$-complex of $\left(H_{\nu}, \Sigma ; \varphi\right)$ is $C^{\varphi}\left(H_{\nu}, \Sigma\right)$ :

$$
0 \rightarrow C_{3}^{\varphi}\left(H_{\nu}, \Sigma\right) \rightarrow C_{2}^{\varphi}\left(H_{\nu}, \Sigma\right) \rightarrow 0
$$

with bases $H_{\nu}$ and $\left(D_{i}^{\nu}\right)_{i}$. Choose the lifts so that

$$
\partial H_{\nu}=\sum_{1 \leq i \leq g}\left(\varphi\left(\nu_{i}^{*}\right)-1\right) D_{i}^{\nu} \in C_{2}^{\varphi}\left(H_{\nu}, \Sigma\right) .
$$

Via the identification $H_{2}^{\varphi}\left(H_{\nu}, \Sigma\right) \simeq L_{\nu}^{\mathbb{F}}$, the basis $\left(\frac{1}{\varphi\left(\nu_{1}^{*}\right)-1} D_{2}^{\nu}, D_{3}^{\nu}, \ldots, D_{g}^{\nu}\right)$ coincides with $h_{\nu}$. Hence $\tau^{\varphi}\left(H_{\nu}, \Sigma ; h_{\nu}\right)=\left[\frac{\partial H_{\nu} \cdot h_{\nu}}{\left(D_{i}^{\nu}\right)_{1 \leq i \leq g}}\right]=1$.

Lemma 6.5. Let $j \in\{1,2,3\}$ and $\nu, \nu^{\prime} \in\{\alpha, \beta, \gamma\}$ be such that $L_{\nu}^{\mathbb{F}} \cap L_{\nu^{\prime}}^{\mathbb{F}} \simeq \partial X_{j}$. There is a symplectic basis $\left(\xi_{i}, \xi_{i}^{*}\right)_{1 \leq i \leq g}$ of $\Sigma$ such that the family $h_{\nu \nu^{\prime}}=\left(\frac{1}{\varphi\left(\xi_{1}^{*}\right)-1} \xi_{2}, \xi_{3}, \ldots, \xi_{k}\right)$ is a basis of $L_{\nu}^{\mathbb{F}} \cap L_{\nu^{\prime}}^{\mathbb{F}}$.

Proof. The surface $\Sigma$ together with the families of curves $\nu$ and $\nu^{\prime}$ is a Heegaard diagram of $\partial X_{j} \simeq \sharp^{k}\left(S^{1} \times S^{2}\right)$. Hence there is a symplectic basis $\left(\xi_{i}, \xi_{i}^{*}\right)_{1 \leq i \leq g}$ such that performing handleslides changes $\left(\nu_{i}\right)_{1 \leq i \leq g}$ into $\left(\xi_{i}\right)_{1 \leq i \leq g}$ and $\left(\nu_{i}^{\prime}\right)_{1 \leq i \leq g}$ into $\left(\xi_{1}, \ldots, \xi_{k}, \xi_{k+1}^{*}, \ldots, \xi_{g}^{*}\right)$. Permuting the indices if necessary, we assume $\varphi\left(\xi_{1}^{*}\right) \neq 1$. Now $\left(\xi_{1}, \ldots, \xi_{g}\right)$ is a system of meridians for $H_{\nu}$ and $\left(\xi_{1}, \ldots, \xi_{k}, \xi_{k+1}^{*}, \ldots, \xi_{g}^{*}\right)$ for $H_{\nu^{\prime}}$. By Lemma 6.3 the families $\left(\frac{1}{\varphi\left(\xi_{1}^{*}\right)-1} \xi_{2}, \xi_{3}, \ldots, \xi_{g}\right)$ and $\left(\frac{1}{\varphi\left(\xi_{1}^{*}\right)-1} \xi_{2}, \xi_{3}, \ldots, \xi_{k}, \xi_{k+1}^{*}, \ldots, \xi_{g}^{*}\right)$ are bases of $L_{\nu}^{\mathbb{F}}$ and $L_{\nu^{\prime}}^{\mathbb{F}}$ respectively.

Lemma 6.6. Via the identification $H_{3}^{\varphi}(X, Y) \simeq\left(L_{\alpha}^{\mathbb{F}} \cap L_{\beta}^{\mathbb{F}}\right) \oplus\left(L_{\beta}^{\mathbb{F}} \cap L_{\gamma}^{\mathbb{F}}\right) \oplus\left(L_{\gamma}^{\mathbb{F}} \cap L_{\alpha}^{\mathbb{F}}\right)$, the family $h_{X, Y}=h_{\alpha \beta} \cdot h_{\beta \gamma} \cdot h_{\gamma \alpha}$ is a homology basis of the pair $(X, Y ; \varphi)$ and we have $\tau^{\varphi}\left(X, Y ; h_{X, Y}\right)=1$. 
Proof. Fix $j \in\{1,2,3\}$ and $\nu, \nu^{\prime} \in\{\alpha, \beta, \gamma\}$ such that $\partial X_{j} \simeq H_{\nu} \cup H_{\nu^{\prime}}$. Let $\left(\xi_{i}, \xi_{i}^{*}\right)_{1 \leq i \leq g}$ be a symplectic basis of $\Sigma$ given by Lemma 6.5. For $i=1, \ldots, g$, let $D_{i}$ be a meridian disk of $H_{\nu}$ with $\partial D_{i}=\xi_{i}$. Similarly let $\Delta_{i}$ be meridians disks of $H_{\nu^{\prime}}$ such that $\partial \Delta_{i}=\xi_{i}$ for $1 \leq i \leq k$ and $\partial \Delta_{i}=\xi_{i}^{*}$ for $k<i \leq g$. The handlebody $X_{j}$ is obtained from $\partial X_{j}$ by adding 3-cells $B_{i}$ such that $\partial B_{i}=D_{i}-\Delta_{i}$ for $1 \leq i \leq k$ and a $4-$ cell $X_{j}$. The associated $\mathbb{F}$-complex is $C^{\varphi}\left(X_{j}, \partial X_{j}\right)$ :

$$
0 \rightarrow C_{4}^{\varphi}\left(X_{j}, \partial X_{j}\right) \rightarrow C_{3}^{\varphi}\left(X_{j}, \partial X_{j}\right) \rightarrow 0
$$

with bases $X_{j}$ and $\left(B_{i}\right)_{1 \leq i \leq k}$. Choose the lifts so that

$$
\partial X_{j}=\sum_{1 \leq i \leq k}\left(\varphi\left(\xi_{i}^{*}\right)-1\right) B_{i} \in C_{3}^{\varphi}\left(X_{j}, \partial X_{j}\right)
$$

Hence $H_{\ell}^{\varphi}\left(X_{j}, \partial X_{j}\right)=0$ if $\ell \neq 3$. Moreover, by Blanchfield duality, $H_{3}\left(X_{j}, \partial X_{j}\right) \simeq$ $H_{1}^{\varphi}\left(X_{j}\right)$. Since $X_{j}$ is obtained from $\partial X_{j}$ by adding 3 and 4-cells, $H_{1}^{\varphi}\left(X_{j}\right) \simeq H_{1}^{\varphi}\left(\partial X_{j}\right)$. Finally, by Lemma 5.4 and Blanchfield duality, $H_{1}^{\varphi}\left(\partial X_{j}\right) \simeq H_{2}^{\varphi}\left(\partial X_{j}\right) \simeq H_{3}^{\varphi}\left(X_{j}, \partial X_{j}\right) \simeq$ $L_{\nu}^{\mathbb{F}} \cap L_{\nu^{\prime}}^{\mathbb{F}}$. A basis of $H_{2}^{\varphi}\left(\partial X_{j}\right)$ is obtained by identifying $\xi_{i}$ with $D_{i} \cup \Delta_{i}$. Its Blanchfield dual basis of $H_{1}^{\varphi}\left(\partial X_{j}\right)$ is $\left(\frac{1}{\varphi\left(\xi_{1}^{*}\right)-1} \xi_{2}^{*}, \xi_{3}^{*}, \ldots, \xi_{k}^{*}\right)$, which is also a basis of $H_{1}^{\varphi}\left(X_{j}\right)$. Its Blanchfield dual basis of $H_{3}\left(X_{j}, \partial X_{j}\right)$ is $\left(\frac{1}{\varphi\left(\xi_{1}^{*}\right)-1} B_{2}, B_{3}, \ldots, B_{k}\right)$ and

$$
\tau^{\varphi}\left(X_{j}, \partial X_{j} ; h_{\nu \nu^{\prime}}\right)=\left[\frac{\partial X_{j} \cdot h_{\nu \nu^{\prime}}}{\left(B_{i}\right)_{1 \leq i \leq k}}\right]=1 .
$$

Conclude with $C^{\varphi}(X, Y) \simeq \oplus_{j=1}^{3} C^{\varphi}\left(X_{j}, \partial X_{j}\right)$.

6.2. Computation of the torsion of $(X ; \varphi)$. In this subsection, we prove Theorem 2.7. Let $h_{X}$ be a homology basis of $(X ; \varphi)$. Let $h_{Y}$ be a homology basis of $Y$, with $h_{Y}^{1}=h_{X}^{1}$ -recall there is a natural identification $H_{1}^{\varphi}(X) \simeq H_{1}^{\varphi}(Y)$. Let $h_{\Sigma}$ be a homology basis of $\Sigma$ as provided by Lemma 6.2. For the pair $(Y, \Sigma)$, fix the homology basis $h_{Y, \Sigma}=h_{\alpha} \cdot h_{\beta} \cdot h_{\gamma}$. For the pair $(X, Y)$, fix the homology basis $h_{X, Y}=h_{\alpha \beta} \cdot h_{\beta \gamma} \cdot h_{\gamma \alpha}$.

Lemma 6.7. The exact sequence in homology associated with the pair $(Y, \Sigma ; \varphi)$ reduces to

$\left(\mathcal{H}_{Y}\right)$

$$
0 \longrightarrow H_{2}^{\varphi}(Y) \longrightarrow H_{2}^{\varphi}(Y, \Sigma) \longrightarrow H_{1}^{\varphi}(\Sigma) \longrightarrow H_{1}^{\varphi}(Y) \longrightarrow 0
$$

and we have $\tau^{\varphi}\left(Y ; h_{Y}\right)=\tau\left(\mathcal{H}_{Y}\right)$.

Proof. The exact sequence of complexes associated with the pair $(Y, \Sigma)$ provides the following equality (see Lemma 3.1):

$$
\tau^{\varphi}\left(Y ; h_{Y}\right)=\tau^{\varphi}\left(\Sigma ; h_{\Sigma}\right) \tau^{\varphi}\left(Y, \Sigma ; h_{Y, \Sigma}\right) \tau\left(\mathcal{H}_{Y}\right) .
$$

The result follows from Lemmas 6.2 and 6.4 .

Lemma 6.8. Let $\mathcal{H}_{X}$ be the exact sequence in homology associated with the pair $(X, Y)$. We have $\tau\left(\mathcal{H}_{X}\right)=\tau\left(\mathcal{H}_{X}^{\prime}\right)$, where $\mathcal{H}_{X}^{\prime}$ is the following part of the sequence $\mathcal{H}_{X}$ itself:

$$
0 \rightarrow H_{3}^{\varphi}(X) \rightarrow H_{3}^{\varphi}(X, Y) \rightarrow H_{2}^{\varphi}(Y) \rightarrow H_{2}^{\varphi}(X) \rightarrow 0 .
$$

Proof. The sequence $\mathcal{H}_{X}$ is composed of $\mathcal{H}_{X}^{\prime}$ and $0 \rightarrow H_{1}^{\varphi}(Y) \rightarrow H_{1}^{\varphi}(X) \rightarrow 0$. 
Proof of Theorem 2.7. Here, $h_{X}$ is the homology basis $h$ of the statement. The exact sequence with coefficients in $\mathbb{F}$ associated with the pair $(X, Y)$ induces the following equality:

$$
\tau^{\varphi}\left(X ; h_{X}\right)=\tau^{\varphi}\left(Y ; h_{Y}\right) \tau^{\varphi}\left(X, Y ; h_{X, Y}\right) \tau\left(\mathcal{H}_{X}\right) .
$$

Hence Lemmas 6.6 and 6.7 give:

$$
\tau^{\varphi}\left(X ; h_{X}\right)=\tau\left(\mathcal{H}_{Y}\right) \tau\left(\mathcal{H}_{X}^{\prime}\right)
$$

By Lemmas 5.2 and 6.7, the sequence $\mathcal{H}_{Y}$ writes:

$$
0 \rightarrow H_{2}^{\varphi}(Y) \rightarrow L_{\alpha}^{\mathbb{F}} \oplus L_{\beta}^{\mathbb{F}} \oplus L_{\gamma}^{\mathbb{F}} \rightarrow H_{1}^{\varphi}(\Sigma) \rightarrow H_{1}^{\varphi}(Y) \rightarrow 0 .
$$

Fixing a basis $s$ for $L_{\alpha}^{\mathbb{F}}+L_{\beta}^{\mathbb{F}}+L_{\gamma}^{\mathbb{F}} \subset H_{1}^{\varphi}(\Sigma)$, we get

$$
\tau\left(\mathcal{H}_{Y}\right)=\left[\frac{h_{Y}^{2} \cdot s}{h_{\alpha} \cdot h_{\beta} \cdot h_{\gamma}}\right]^{-1}\left[\frac{s \cdot h_{X}^{1}}{h_{\Sigma}}\right] \text {. }
$$

By Lemmas 5.4 and 6.8 , the sequence $\mathcal{H}_{X}^{\prime}$ writes:

$$
0 \rightarrow H_{3}^{\varphi}(X) \rightarrow\left(L_{\alpha}^{\mathbb{F}} \cap L_{\beta}^{\mathbb{F}}\right) \oplus\left(L_{\beta}^{\mathbb{F}} \cap L_{\gamma}^{\mathbb{F}}\right) \oplus\left(L_{\gamma}^{\mathbb{F}} \cap L_{\alpha}^{\mathbb{F}}\right) \stackrel{\zeta}{\longrightarrow} H_{2}^{\varphi}(Y) \rightarrow H_{2}^{\varphi}(X) \rightarrow 0 .
$$

Fixing a basis $t$ for $\operatorname{Im}(\zeta) \subset H_{2}^{\varphi}(Y)$, we get

$$
\tau\left(\mathcal{H}_{X}^{\prime}\right)=\left[\frac{h_{X}^{3} \cdot t}{h_{\alpha \beta} \cdot h_{\beta \gamma} \cdot h_{\gamma \alpha}}\right]\left[\frac{t \cdot h_{X}^{2}}{h_{Y}^{2}}\right]^{-1} .
$$

Fixing the complex basis $c=\left(h_{\alpha \beta} \cdot h_{\beta \gamma} \cdot h_{\gamma \alpha}, h_{\alpha} \cdot h_{\beta} \cdot h_{\gamma}, h_{\Sigma}\right)$ for the complex $C^{\mathbb{F}}$, we have:

$$
\tau\left(C^{\mathbb{F}} ; c, h_{X}\right)=\left[\frac{h_{X}^{3} \cdot t}{h_{\alpha \beta} \cdot h_{\beta \gamma} \cdot h_{\gamma \alpha}}\right]\left[\frac{t \cdot h_{X}^{2} \cdot s}{h_{\alpha} \cdot h_{\beta} \cdot h_{\gamma}}\right]^{-1}\left[\frac{s \cdot h_{X}^{1}}{h_{\Sigma}}\right],
$$

so that we get the desired equality.

\section{Computation of $\tau^{\varphi}(X)$ Via $(\widehat{X}, \star)$}

In this section, we compute the torsion of $(\widehat{X}, \star ; \varphi)$ and relate it to the torsion of $(X ; \varphi)$. For $\nu \in\{\alpha, \beta, \gamma\}$, let $\widehat{L}_{\nu}^{\mathbb{F}}$ be the subspace of $H_{1}^{\varphi}(\widehat{\Sigma}, \star ; \mathbb{F})$ generated by the homology classes of the curves $\nu_{i}$.

Lemma 7.1. The $\Lambda$-modules $H_{1}^{\varphi}(\widehat{\Sigma}, \star ; \Lambda), \widehat{L}_{\nu}^{\Lambda}$ and $\widehat{L}_{\nu}^{\Lambda} \cap \widehat{L}_{\nu^{\prime}}^{\Lambda}$ are free $\Lambda$-modules of rank $2 g, g$ and $k$ respectively. Moreover, $H_{1}^{\varphi}(\widehat{\Sigma}, \star ; \mathbb{F})=H_{1}^{\varphi}(\widehat{\Sigma}, \star ; \Lambda) \otimes_{\Lambda} \mathbb{F}, \widehat{L}_{\nu}^{\mathbb{F}}=\widehat{L}_{\nu}^{\Lambda} \otimes_{\Lambda} \mathbb{F}$ and $\widehat{L}_{\nu}^{\mathbb{F}} \cap \widehat{L}_{\nu^{\prime}}^{\mathbb{F}}=\left(\widehat{L}_{\nu}^{\Lambda} \cap \widehat{L}_{\nu^{\prime}}^{\Lambda}\right) \otimes_{\Lambda} \mathbb{F}$.

Proof. Set $R=\Lambda$ or $\mathbb{F}$. Fix $\nu \in\{\alpha, \beta, \gamma\}$. Let $\left(\nu_{i}^{*}\right)_{1 \leq i \leq g}$ be a family of curves on $\widehat{\Sigma}$ such that $\left(\nu_{i}, \nu_{i}^{*}\right)_{1 \leq i \leq g}$ is a symplectic basis for $\Sigma$. Assume the removed ball $B$ is such that $\partial D=\prod_{i=1}^{g}\left[\nu_{i}, \nu_{i}^{*}\right]$. Consider a CW-decomposition of $\widehat{\Sigma}$ with one 0-cell $\star$, one 2-cell $\widehat{\Sigma}$ and 1-cells $\nu_{i}, \nu_{i}^{*}$ and $\partial D$. The associated $\mathbb{F}$-complex is $C^{\varphi}(\widehat{\Sigma}, \star)$ :

$$
0 \rightarrow C_{2}^{\varphi}(\widehat{\Sigma}, \star) \rightarrow C_{1}^{\varphi}(\widehat{\Sigma}, \star) \rightarrow 0
$$


Choose a lift of $\widehat{\Sigma}$ such that:

$$
\partial \widehat{\Sigma}=-\partial D+\sum_{1 \leq i \leq g}\left(\varphi\left(\nu_{i}^{*}\right)-1\right) \nu_{i} \in C_{1}^{\varphi}(\widehat{\Sigma}, \star)
$$

The only non-trivial homology $R$-module of $(\widehat{\Sigma}, \star)$ is $H_{1}^{\varphi}(\widehat{\Sigma}, \star ; R) \simeq R^{2 g}$ generated by $\nu_{i}$ and $\nu_{i}^{*}$ for $i=1, \ldots, g$. Since $\widehat{L}_{\nu}^{R}$ is the submodule of $H_{1}^{\varphi}(\widehat{\Sigma}, \star ; R)$ generated by the $\nu_{i}$, we get $\widehat{L}_{\nu}^{R} \simeq R^{g}$.

A similar computation can be done for $(\widehat{\Sigma}, \star)$ from any symplectic basis for $\Sigma$. As in Lemma 6.5, if $\nu$ and $\nu^{\prime}$ are distinct, there exists a symplectic basis $\left(\xi_{i}, \xi_{i}^{*}\right)_{1 \leq i \leq g}$ for $\Sigma$ such that $\widehat{L}_{\nu}^{R} \cap \widehat{L}_{\nu^{\prime}}^{R} \simeq R^{k}$ freely generated by $\left(\xi_{i}\right)_{1 \leq i \leq k}$.

If $\Gamma$ is a free $\Lambda$-module, a $\Lambda$-basis of $\Gamma \otimes_{\Lambda} \mathbb{F}$ is a basis $b \otimes 1$ where $b$ is a basis of $\Gamma$.

Theorem 7.2. The twisted homology of $(\widehat{X}, \star)$ canonically identifies with the homology of the following $\mathbb{F}$-complex $\widehat{C}^{\mathbb{F}}$ :

$$
0 \rightarrow\left(\widehat{L}_{\alpha}^{\mathbb{F}} \cap \widehat{L}_{\beta}^{\mathbb{F}}\right) \oplus\left(\widehat{L}_{\beta}^{\mathbb{F}} \cap \widehat{L}_{\gamma}^{\mathbb{F}}\right) \oplus\left(\widehat{L}_{\gamma}^{\mathbb{F}} \cap \widehat{L}_{\alpha}^{\mathbb{F}}\right) \stackrel{\zeta}{\longrightarrow} \widehat{L}_{\alpha}^{\mathbb{F}} \oplus \widehat{L}_{\beta}^{\mathbb{F}} \oplus \widehat{L}_{\gamma}^{\mathbb{F}} \stackrel{\iota}{\longrightarrow} H_{1}^{\varphi}(\widehat{\Sigma}, \star ; \mathbb{F}) \rightarrow 0,
$$

where $\zeta(x, y, z)=(x-z, y-x, z-y)$ and $\iota$ is defined by the inclusions $\widehat{L}_{\nu}^{\mathbb{F}} \hookrightarrow H_{1}^{\varphi}(\widehat{\Sigma}, \star ; \mathbb{F})$. Moreover, for any complex $\Lambda$-basis $\widehat{c}$ for $\widehat{C}^{\mathbb{F}}$ and any homology basis $\widehat{h}$ for $(\widehat{X}, \star)$ and $\widehat{C}^{\mathbb{F}}$, we have:

$$
\tau^{\varphi}(\widehat{X}, \star ; \widehat{h})=\tau\left(\widehat{C}^{\mathbb{F}} ; \widehat{c}, \widehat{h}\right) \quad \text { in } \mathbb{F} / \Lambda^{*}
$$

Proof. Using Lemma 7.1, the whole sections [5 and [6 adapt to the setting of $(\widehat{X}, \star)$, providing the result. The independance with respect to the choice of a $\Lambda$-bases over $\mathbb{F}$ for $\widehat{C}^{\mathbb{F}}$ is due to the fact that a change of such bases modifies the torsion by an element of $\Lambda^{*}$.

Lemma 7.3. For all $\ell \neq 1,2,3$, one has $H_{\ell}^{\varphi}(X ; \mathbb{F})=H_{\ell}^{\varphi}(X, \star)=H_{\ell}^{\varphi}(\widehat{X}, \star ; \mathbb{F})=0$. Moreover, there are natural identifications of $\mathbb{F}$-vector spaces

$$
H_{1}^{\varphi}(X, \star) \simeq H_{1}^{\varphi}(\widehat{X}, \star), H_{2}^{\varphi}(X) \simeq H_{2}^{\varphi}(X, \star) \simeq H_{2}^{\varphi}(\widehat{X}, \star) \text { and } H_{3}^{\varphi}(X) \simeq H_{3}^{\varphi}(X, \star)
$$

and short exact sequences of $\mathbb{F}$-vector spaces

$$
0 \rightarrow H_{1}^{\varphi}(X) \rightarrow H_{1}^{\varphi}(\widehat{X}, \star) \rightarrow H_{0}^{\varphi}(\star) \rightarrow 0,0 \rightarrow H_{4}^{\varphi}(B, \partial B) \rightarrow H_{3}^{\varphi}(\widehat{X}, \star) \rightarrow H_{3}^{\varphi}(X) \rightarrow 0 .
$$

Proof. The result follows from the exact sequence in homology of the pair $(X, \star)$ and from the exact sequence in homology of the triple $(X, \widehat{X}, \star)$ combined with the excision equivalence $(X, \widehat{X}) \simeq(B, \partial B)$.

Proposition 7.4. Let $h$ be a homology basis of $X$. Let $u \in H_{1}(\widehat{X}, \star)$ satisfy $\varphi(u) \neq 1$. Then $\widehat{h}=\left(\partial B . h^{3}, h^{2}, h^{1} \cdot u\right)$ is a homology basis of $(\widehat{X}, \star)$ and

$$
(\varphi(u)-1) \tau^{\varphi}(X ; h)=\tau^{\varphi}(\widehat{X}, \star ; \widehat{h}) .
$$

Proof. Thanks to Lemma 7.3, $\widehat{h}$ is a homology basis for $(\widehat{X}, \star)$ and $\hat{h}=\left(h^{3}, h^{2}, h^{1} . u\right)$ is a homology basis for $(X, \star)$. The short exact sequences of complexes $0 \rightarrow C^{\varphi}(\star) \rightarrow$ $C^{\varphi}(X) \rightarrow C^{\varphi}(X, \star) \rightarrow 0$ and $0 \rightarrow C^{\varphi}(\widehat{X}, \star) \rightarrow C^{\varphi}(X, \star) \rightarrow C^{\varphi}(B, \partial B) \rightarrow 0$ provide

$$
\tau^{\varphi}(X ; h)=\tau^{\varphi}(\star ; \star) \tau^{\varphi}(X, \star ; \bar{h}) \tau\left(\mathcal{S}_{1}\right)
$$


TORSIONS AND INTERSECTION FORMS OF 4-MANIFOLDS FROM TRISECTION DIAGRAMS 15

and

$$
\tau^{\varphi}(X, \star ; \bar{h})=\tau^{\varphi}(\widehat{X}, \star ; \widehat{h}) \tau^{\varphi}(B, \partial B ; B) \tau\left(\mathcal{S}_{2}\right)
$$

where $\mathcal{S}_{1}$ and $\mathcal{S}_{2}$ are the associated exact sequences in homology. One easily checks that $\tau^{\varphi}(\star ; \star)=1$ and $\tau^{\varphi}(B, \partial B ; B)=1$, so that:

$$
\tau^{\varphi}(X ; h)=\tau^{\varphi}(\widehat{X}, \star ; \widehat{h}) \tau\left(\mathcal{S}_{1}\right) \tau\left(\mathcal{S}_{2}\right) .
$$

A straightforward computation shows that $\tau\left(\mathcal{S}_{1}\right)=(\varphi(u)-1)^{-1}$ and $\tau\left(\mathcal{S}_{2}\right)=1$.

\section{INTERSECTION FORMS}

In this section, we prove the results on intersection forms. Along the proofs, we give interpretations of the modules of the complexes $C$ and $C^{\mathbb{F}}$ as modules of chains.

We first reprove the expression of the intersection form on $H_{2}(X ; \mathbb{Z})$ given in [FKSZ18] with our approach. Following Wall Wal69, define the symmetric form

$$
\lambda: \frac{L_{\alpha} \cap\left(L_{\beta}+L_{\gamma}\right)}{\left(L_{\alpha} \cap L_{\beta}\right)+\left(L_{\alpha} \cap L_{\gamma}\right)} \times \frac{L_{\alpha} \cap\left(L_{\beta}+L_{\gamma}\right)}{\left(L_{\alpha} \cap L_{\beta}\right)+\left(L_{\alpha} \cap L_{\gamma}\right)} \longrightarrow \mathbb{Z}
$$

as follows. For $a, a^{\prime} \in L_{\alpha} \cap\left(L_{\beta}+L_{\gamma}\right)$ and $b \in L_{\beta}, c \in L_{\gamma}$ such that $a+b+c=0$, set

$$
\lambda\left(a, a^{\prime}\right):=\left\langle c, a^{\prime}\right\rangle_{\Sigma} \text {. }
$$

Proposition 8.1 ([FKSZ18] $)$. Let $\langle\cdot, \cdot\rangle_{X}$ be the intersection form of $X$. There is an isomorphism

$$
\left(H_{2}(X ; \mathbb{Z}) ;\langle\cdot, \cdot\rangle_{X}\right) \simeq\left(\frac{L_{\alpha} \cap\left(L_{\beta}+L_{\gamma}\right)}{\left(L_{\alpha} \cap L_{\beta}\right)+\left(L_{\alpha} \cap L_{\gamma}\right)} ; \lambda\right) .
$$

Proof. By Theorem 2.1,

$$
H_{2}(X) \simeq \frac{\operatorname{ker}\left(L_{\alpha} \oplus L_{\beta} \oplus L_{\gamma} \rightarrow H_{1}(\Sigma ; \mathbb{Z})\right)}{\left(L_{\alpha} \cap L_{\beta}\right) \oplus\left(L_{\beta} \cap L_{\gamma}\right) \oplus\left(L_{\gamma} \cap L_{\alpha}\right)} \simeq \frac{L_{\alpha} \cap\left(L_{\beta}+L_{\gamma}\right)}{\left(L_{\alpha} \cap L_{\beta}\right)+\left(L_{\alpha} \cap L_{\gamma}\right)} .
$$

Following the trisection, $X$ is built from $D^{2} \times \Sigma$ by adding $2,3,4$-cells attached to $S^{1} \times \Sigma$, see Section 3.4. Let $p_{0}, p_{\alpha}, p_{\beta}, p_{\gamma}$ be the points in $D^{2}$ defined as in Figure 2. Given $\mu=\left(\mu_{\alpha}, \mu_{\beta}, \mu_{\gamma}\right)$ in $\operatorname{ker}\left(L_{\alpha} \oplus L_{\beta} \oplus L_{\gamma} \rightarrow H_{1}(\Sigma)\right)$ and a point $q \neq p_{0} \in \operatorname{Int}\left(D^{2}\right)$, we construct a 2-cycle $S_{q}(\mu) \in C_{2}(X)$ as follows. For all $\nu \in\{\alpha, \beta, \gamma\}$, write $\mu_{\nu}$ as a linear combination of the $\nu_{i}$ and define $D_{\nu}(\mu)$ as the corresponding disjoint union of meridian disks bounded by parallel copies of the $\left\{p_{\nu}\right\} \times \nu_{i}$. Then define

$$
S_{q}(\mu):=S_{\alpha}+S_{\beta}+S_{\gamma}+T(\mu),
$$

where:

- $S_{\nu}=D_{\nu}(\mu) \cup\left(\left[p_{\nu}, q\right] \times \mu_{\nu}\right)$,

- $T(\mu)$ is a 2-chain with support contained in $\{q\} \times \Sigma$ with $\partial T(\mu)=\mu_{\alpha}+\mu_{\beta}+\mu_{\gamma}$. Let now $\mu=\left(\mu_{\alpha}, \mu_{\beta}, \mu_{\gamma}\right)$ and $\mu^{\prime}=\left(\mu_{\alpha}^{\prime}, \mu_{\beta}^{\prime}, \mu_{\gamma}^{\prime}\right)$ be in $\operatorname{ker}\left(L_{\alpha} \oplus L_{\beta} \oplus L_{\gamma} \rightarrow H_{1}(\Sigma)\right)$. Fix $q$ and $q^{\prime}$ in $\operatorname{Int}\left(D^{2}\right)$ such that $p_{0}=\left(p_{\gamma}, q\right) \cap\left(p_{\alpha}, q^{\prime}\right)$. The 2-cycles $S_{q}(\mu)$ and $S_{q^{\prime}}\left(\mu^{\prime}\right)$ intersect transversally in $\left\{p_{0}\right\} \times \Sigma$ and

$$
\left\langle S_{q}(\mu), S_{q^{\prime}}\left(\mu^{\prime}\right)\right\rangle_{X}=\left\langle\mu_{\gamma}, \mu_{\alpha}^{\prime}\right\rangle_{\Sigma}=\lambda\left(\mu, \mu^{\prime}\right),
$$

where we assume that $D^{2}$ is oriented by the oriented basis $\left(p_{0} \vec{p}_{\alpha}, p_{0} \vec{p}_{\gamma}\right)$. 

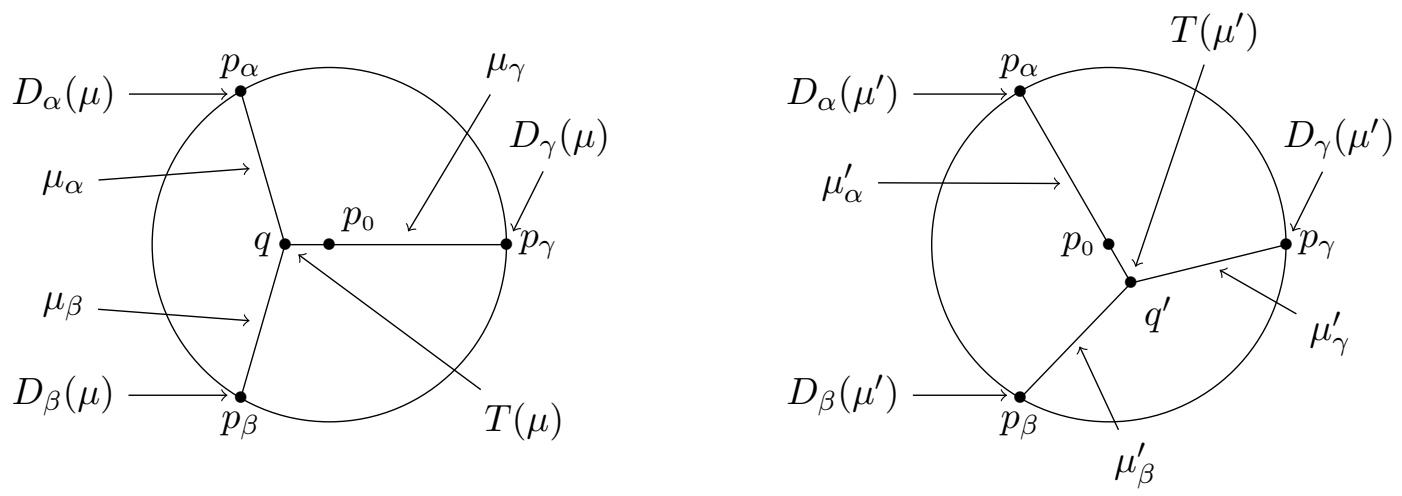

Figure 3. The 2-cycles $S$ and $S^{\prime}$.

We now prove Theorem 2.5, which is the analogue in the twisted setting of Proposition 8.1 .

Proof of Theorem 2.5. All the curves of the families $\alpha, \beta, \gamma$ have their homology classes in $\operatorname{ker}(\varphi)$, so that they lift as loops. Moreover, the meridian disks and the paths of Figure 3 drawn on $D^{2}$ are contractible, thus they also lift as disks and paths. Hence the result follows from the very same argument as in Proposition 8.1,

We turn to the intersection form on $H_{1}(X ; \mathbb{Z}) \times H_{3}(X ; \mathbb{Z})$.

Proposition 8.2. There is an isomorphism

$$
\left(H_{1}(X ; \mathbb{Z}) \times H_{3}(X ; \mathbb{Z}) ;\langle\cdot, \cdot\rangle_{X}\right) \cong\left(\frac{H_{1}(\Sigma)}{L_{\alpha}+L_{\beta}+L_{\gamma}} \times\left(L_{\alpha} \cap L_{\beta} \cap L_{\gamma}\right) ;\langle\cdot, \cdot\rangle_{\Sigma}\right) .
$$

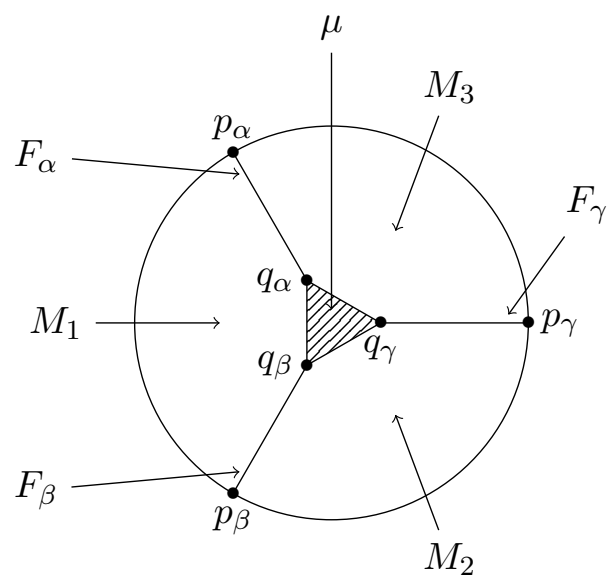

Figure 4. The 3-cycle $M$ associated to $\mu$. 
Proof of Proposition 8.2. Let $\mu \in L_{\alpha} \cap L_{\beta} \cap L_{\gamma}$. By a slight abuse of notation, we use the same letter $\mu$ for a representative of it on $\Sigma$. We will construct a 3-cycle $M$ associated with $\mu$ that intersects $\Sigma$ along $\mu$. Once again, we view $X$ as reconstructed from the trisection diagram. Let $q_{\alpha}, q_{\beta}, q_{\gamma}$ be the points on $D^{2}$ represented in Figure 4 and let $V$ be the hatched triangle they define. We will complete the 3-chain $M_{0}=V \times \mu$ into a 3 cycle. For each $\nu \in\{\alpha, \beta, \gamma\}, \mu$ bounds a surface $F_{\nu}$ properly embedded in $H_{\nu}$. For $i, \nu, \nu^{\prime}$ such that $\partial X_{i}=H_{\nu} \cup H_{\nu^{\prime}}$, since $X_{i} \cong\left(X_{i} \backslash(\operatorname{Int}(V) \times \Sigma)\right)$ has trivial second homology, the closed surface $F_{\nu} \cup\left(\left[q_{\nu}, q_{\nu^{\prime}}\right] \times \mu\right) \cup F_{\nu^{\prime}}$ bounds a 3-cycle $M_{i} \subset\left(X_{i} \backslash(\operatorname{Int}(V) \times \Sigma)\right)$. Finally, $M=\sum_{0 \leq i \leq 3} M_{i}$ is a 3-cycle associated with $\mu$. Then, for any $\mu^{\prime} \in H_{1}(\Sigma)$, we have $\left\langle\mu^{\prime}, M\right\rangle_{X}=\left\langle\mu^{\prime}, \mu_{\alpha}\right\rangle_{\Sigma}=\left\langle\mu^{\prime}, \mu\right\rangle_{\Sigma}$.

A similar proof yields Proposition 2.6.

\section{EXAMPLES}

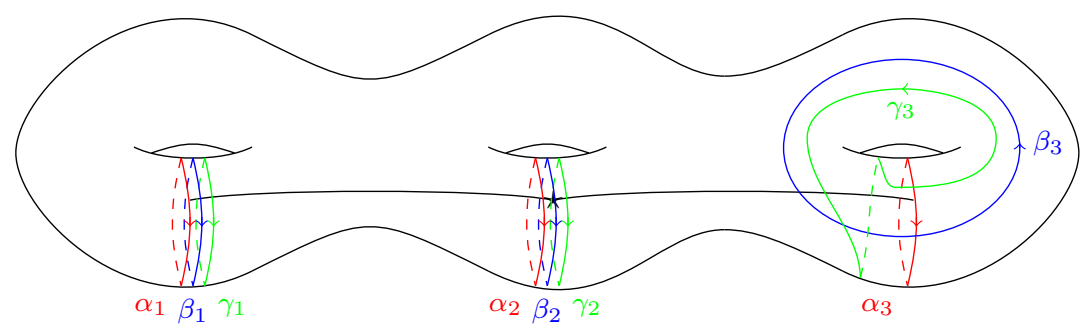

FiguRE 5. A trisection diagram for $\left(S^{1} \times S^{2}\right) \sharp\left(S^{1} \times S^{2}\right) \sharp \mathbb{C} P^{2}$

Example 1. The trisection diagram $(\Sigma ; \alpha, \beta, \gamma)$ in Figure 5 represents the 4 -manifold $X=\left(S^{1} \times S^{3}\right) \sharp\left(S^{1} \times S^{3}\right) \sharp \mathbb{C} P^{2}$. The black paths fix a choice of a representative in $\pi_{1}(\Sigma, \star)$ of each loop. Let $x_{i}, y_{i}$ for $i \in\{1,2,3\}$ be the generators of $\pi_{1}(\Sigma, \star)$ represented in Figure 6. Their homology classes provide a symplectic basis of $H_{1}(\Sigma ; \mathbb{Z})$. Note that the family $\left(x_{i}, y_{i}\right)_{1 \leq i \leq 3}$ is not a symplectic basis for $\Sigma$ as in Definition 6.1, although it could easily be modified to get such a basis. The following relations hold in $\pi_{1}(\Sigma, \star)$ : $\alpha_{1}=\beta_{1}=\gamma_{1}=x_{1}, \alpha_{2}=\beta_{2}=\gamma_{2}=x_{2}, \alpha_{3}=x_{3}, \beta_{3}=y_{3}$ and $\gamma_{3}=x_{3} y_{3}$.

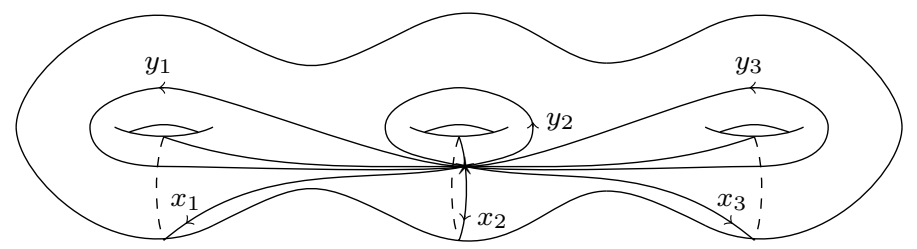

Figure 6 . A basis of $H_{1}(\Sigma ; \mathbb{Z})$ with curves based at $\star$ 
Setting $L=\left\langle x_{1}, x_{2}\right\rangle \subset H_{1}(\Sigma ; \mathbb{Z})$, we get

$$
L_{\alpha}=L \oplus\left\langle x_{3}\right\rangle, L_{\beta}=L \oplus\left\langle y_{3}\right\rangle \text { and } L_{\gamma}=L \oplus\left\langle x_{3}+y_{3}\right\rangle \text {. }
$$

Hence, by Theorem 2.1;

- $H_{1}(X ; \mathbb{Z}) \simeq \mathbb{Z}^{2}$ is generated by $y_{1}$ and $y_{2}$

- $H_{2}(X ; \mathbb{Z}) \simeq \mathbb{Z}$ is generated by $x_{3}$

- $H_{3}(X ; \mathbb{Z}) \simeq \mathbb{Z}^{2}$ is generated by $x_{1}$ and $x_{2}$.

In these bases, the matrix of the intersection form on $H_{2}(X ; \mathbb{Z})$ is $(1)$ and the matrix of the form on $H_{1}(X ; \mathbb{Z}) \times H_{3}(X ; \mathbb{Z})$ is $\left(\begin{array}{cc}-1 & 0 \\ 0 & -1\end{array}\right)$, see Proposition 8.1 and Proposition 8.2 .

Now let $G \simeq \mathbb{Z}^{2}$ be the free abelian (multiplicative) group of rank 2 generated by $t_{1}$ and $t_{2}$. Let $\varphi: H_{1}(X ; \mathbb{Z}) \rightarrow G$ be defined by $\varphi\left(y_{1}\right)=t_{1}$ and $\varphi\left(y_{2}\right)=t_{2}$. The following relations hold in $H_{1}^{\varphi}(\Sigma ; R)$, assuming the lifts of the curves all start at the same lift of the point $\star: \alpha_{1}=\beta_{1}=\gamma_{1}=x_{1}, \alpha_{2}=\beta_{2}=\gamma_{2}=x_{2}, \alpha_{3}=x_{3}, \beta_{3}=y_{3}$ and $\gamma_{3}=x_{3}+y_{3}$.

In the cellular decomposition of $\Sigma$ given by Figure 6, the only 2-cell has boundary $\partial \Sigma=\left[x_{1}, y_{1}\right] y_{2}^{-1}\left[y_{3}^{-1}, x_{3}\right] x_{2} y_{2} x_{2}^{-1}$. This provides a single relation in $H_{1}^{\varphi}(\Sigma ; R)$ :

$$
r=\left(1-t_{1}\right) x_{1}+\left(t_{2}^{-1}-1\right) x_{2} .
$$

Setting $L^{R}=\left\langle x_{1}, x_{2}\right\rangle /\langle r\rangle \subset H_{1}^{\varphi}(\Sigma ; R)$, we get

$$
H_{1}^{\varphi}(\Sigma ; R)=L^{R} \oplus\left\langle\left(1-t_{2}\right) y_{1}+\left(t_{1}-1\right) y_{2}, x_{3}, y_{3}\right\rangle
$$

and

$$
L_{\alpha}^{R}=L^{R} \oplus\left\langle x_{3}\right\rangle, L_{\beta}^{R}=L^{R} \oplus\left\langle y_{3}\right\rangle \text { and } L_{\gamma}^{R}=L^{R} \oplus\left\langle x_{3}+y_{3}\right\rangle .
$$

Hence by Theorem 2.3 .

- $H_{1}^{\varphi}(X ; R) \simeq R$ is generated by $\left(1-t_{2}\right) y_{1}+\left(t_{1}-1\right) y_{2}$

- $H_{2}^{\varphi}(X ; R) \simeq R$ is generated by $x_{3}$,

- $H_{3}^{\varphi}(X ; R) \simeq L^{R}$ and $H_{3}^{\varphi}(X ; \mathbb{F}) \simeq \mathbb{F}$ is generated by $x_{1}$.

In these generators, the intersection form on $H_{2}^{\varphi}(X ; \mathbb{F}) \simeq \mathbb{F}$ is given by 1 and the intersection form on $H_{1}^{\varphi}(X ; \mathbb{F}) \times H_{3}^{\varphi}(X ; \mathbb{F}) \simeq \mathbb{F} \times \mathbb{F}$ is given by $t_{1}\left(t_{2}-1\right)$, see Theorem 2.5 and Proposition 2.6.

We end with the computation of the torsion. Fix the homology basis $h=\left(h_{3}, h_{2}, h_{1}\right)$ with $h_{3}=x_{1}, h_{2}=x_{3}, h_{1}=\left(1-t_{2}\right) y_{1}+\left(t_{1}-1\right) y_{2}$. We compute the torsion $\tau^{\varphi}(X ; h) \in$ $\mathbb{F} / \Lambda^{*}$. Set $u=y_{1}$. By Proposition [7.4, $\tau^{\varphi}(X ; h)=\left(t_{1}-1\right)^{-1} \tau^{\varphi}(\widehat{X}, \star ; \widehat{h})$ where $\widehat{h}=$ $\left(\widehat{h}_{3}, \widehat{h}_{2}, \widehat{h}_{1}\right)$ and $\widehat{h}_{3}=\left(r, h_{3}\right), \widehat{h}_{2}=h_{2}, \widehat{h}_{1}=\left(h_{1}, u\right)$. By Theorem $7.2, \tau^{\varphi}(\widehat{X}, \star ; \widehat{h})$ equals the torsion $\tau\left(\widehat{C}^{\mathbb{F}} ; \widehat{c}, \widehat{h}\right)$ of the complex $\widehat{C}^{\mathbb{F}}$ :

$$
0 \rightarrow \widehat{L}^{\mathbb{F}} \oplus \widehat{L}^{\mathbb{F}} \oplus \widehat{L}^{\mathbb{F}} \stackrel{\zeta}{\longrightarrow} \widehat{L}_{\alpha}^{\mathbb{F}} \oplus \widehat{L}_{\beta}^{\mathbb{F}} \oplus \widehat{L}_{\gamma}^{\mathbb{F}} \stackrel{\iota}{\longrightarrow} H_{1}^{\varphi}(\widehat{\Sigma}, \star ; \mathbb{F}) \rightarrow 0
$$


TORSIONS AND INTERSECTION FORMS OF 4-MANIFOLDS FROM TRISECTION DIAGRAMS 19 where $\widehat{c}$ is a $\Lambda$-basis over $\mathbb{F}$ and $\widehat{L}^{\mathbb{F}}=\left\langle x_{1}, x_{2}\right\rangle \subset H_{1}^{\varphi}(\widehat{\Sigma}, \star ; \mathbb{F})$. Define $\widehat{c}=\left(\widehat{c}_{3}, \widehat{c}_{2}, \widehat{c}_{1}\right)$ by

$$
\begin{array}{lr}
\widehat{c}_{3}=\left(\left(x_{1}, 0,0\right),\left(x_{2}, 0,0\right),\left(0, x_{1}, 0\right),\left(0, x_{2}, 0\right),\left(0,0, x_{1}\right),\left(0,0, x_{2}\right)\right), \\
\widehat{c}_{2}=\left(\left(x_{1}, 0,0\right),\left(x_{2}, 0,0\right),\left(x_{3}, 0,0\right),\left(0, x_{1}, 0\right),\left(0, x_{2}, 0\right),\left(0, y_{3}, 0\right),\left(0,0, x_{1}\right),\left(0,0, x_{2}\right),\right. \\
\widehat{c}_{1}=\left(x_{1}, x_{2}, x_{3}, y_{1}, y_{2}, y_{3}\right) .
\end{array}
$$

Also fix the following bases of $\operatorname{Im}(\zeta)$ and $\operatorname{Im}(\iota)$ :

$$
\begin{aligned}
& \widehat{b}_{2}=\left(\left(x_{1},-x_{1}, 0\right),\left(x_{2},-x_{2}, 0\right),\left(0, x_{1},-x_{1}\right),\left(0, x_{2},-x_{2}\right)\right), \\
& \widehat{b}_{1}=\left(x_{1}, x_{2}, x_{3}, y_{3}\right) .
\end{aligned}
$$

Lift the latter two bases to get the following independant families in $\widehat{L}^{\mathbb{F}} \oplus \widehat{L}^{\mathbb{F}} \oplus \widehat{L}^{\mathbb{F}}$ and $\widehat{L}_{\alpha}^{\mathbb{F}} \oplus \widehat{L}_{\beta}^{\mathbb{F}} \oplus \widehat{L}_{\gamma}^{\mathbb{F}}$ :

$$
\begin{aligned}
& \bar{b}_{2}=\left(\left(x_{1}, 0,0\right),\left(x_{2}, 0,0\right),\left(0, x_{1}, 0\right),\left(0, x_{2}, 0\right)\right), \\
& \bar{b}_{1}=\left(\left(x_{1}, 0,0\right),\left(x_{2}, 0,0\right),\left(x_{3}, 0,0\right),\left(0, y_{3}, 0\right)\right) .
\end{aligned}
$$

Now, by definition of the torsion:

$$
\tau\left(\widehat{C}^{\mathbb{F}} ; \widehat{c}, \widehat{h}\right)=\left[\frac{\widehat{h}_{3} \cdot \bar{b}_{2}}{\widehat{c}_{3}}\right] \cdot\left[\frac{\widehat{b}_{2} \cdot \widehat{h}_{2} \cdot \bar{b}_{1}}{\widehat{c}_{2}}\right]^{-1}\left[\frac{\widehat{b}_{1} \cdot \widehat{h}_{1}}{\widehat{c}_{1}}\right] .
$$

A straightforward computation gives $\tau^{\varphi}(X ; h)=1-t_{2} \in \mathbb{F} / \Lambda^{*}$.

Example 2. The trisection diagram $(\Sigma ; \alpha, \beta, \gamma)$ in Figure 7 represents the 4 -manifold $X=S^{1} \times L(3,1)$ product of a circle with the Lens space $L(3,1)$, see [Koe17, Figure 10]. Generators $x_{i}, y_{i}$ of $\pi_{1}(\Sigma, \star)$, with $i \in\{1, \ldots, 4\}$, are given in Figure 8 , Their homology classes provide a symplectic basis of $H_{1}(\Sigma ; \mathbb{Z})$. The following relations hold in $\pi_{1}(\Sigma, \star)$ :

$$
\begin{array}{lll}
\alpha_{1}=x_{1} & \beta_{1}=x_{2} & \gamma_{1}=y_{1} \\
\alpha_{2}=y_{4}^{-1} x_{4} y_{4} x_{4}^{-1} y_{2}^{-1} x_{2} y_{2} & \beta_{2}=x_{3}^{-1} y_{1}^{-1} x_{1} y_{1} & \gamma_{2}=x_{3} y_{3}^{-1} x_{3}^{-1} y_{3} x_{2} \\
\alpha_{3}=y_{2}^{-1} y_{3}^{-1} x_{3}^{-1} y_{3} y_{2} x_{4} & \beta_{3}=y_{4}^{-1} x_{4}^{-1} y_{4} x_{1} & \gamma_{3}=y_{3}^{-1} x_{3} y_{3}^{-2} \\
\alpha_{4}=y_{2}^{-1} y_{3} y_{2} y_{4} & \beta_{4}=y_{1} y_{3} y_{4} & \gamma_{4}=x_{4} y_{4}^{3}
\end{array}
$$

We obtain in $H_{1}(\Sigma ; \mathbb{Z})$ :

$$
\begin{array}{lll}
\alpha_{1}=x_{1} & \beta_{1}=x_{2} & \gamma_{1}=y_{1} \\
\alpha_{2}=x_{2} & \beta_{2}=x_{1}-x_{3} & \gamma_{2}=x_{2} \\
\alpha_{3}=-x_{3}+x_{4} & \beta_{3}=x_{1}-x_{4} & \gamma_{3}=x_{3}-3 y_{3} \\
\alpha_{4}=y_{3}+y_{4} & \beta_{4}=y_{1}+y_{3}+y_{4} & \gamma_{4}=x_{4}+3 y_{4}
\end{array}
$$

Hence, by Theorem 2.1

- $H_{1}(X ; \mathbb{Z}) \simeq \mathbb{Z} \oplus \frac{\mathbb{Z}}{3 \mathbb{Z}}$ with the first summand generated by $y_{2}$ and the second by $y_{3}$,

- $H_{2}(X ; \mathbb{Z}) \simeq \frac{\mathbb{Z}}{3 \mathbb{Z}}$ is generated by $y_{3}+y_{4}$,

- $H_{3}(X ; \mathbb{Z}) \simeq \mathbb{Z}$ is generated by $x_{2}$. 


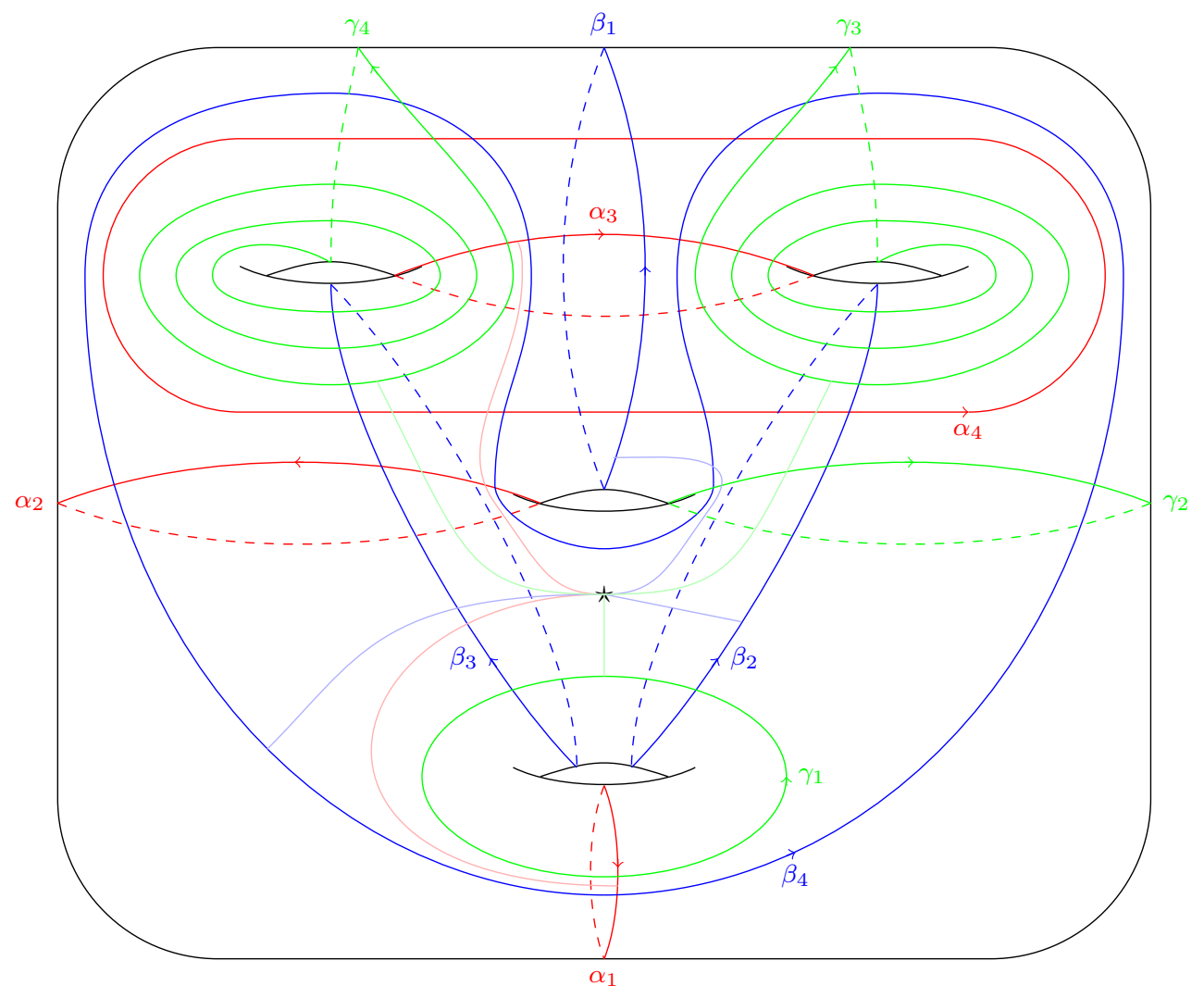

FiguRE 7. A trisection diagram for $S^{1} \times L(3,1)$

In these bases, the intersection form on $H_{1}(X ; \mathbb{Z}) \times H_{3}(X ; \mathbb{Z})$ is given by $\left\langle y_{2}, x_{2}\right\rangle=-1$.

Now let $G \simeq \mathbb{Z}$ be the multiplicative group generated by $t$. Let $\varphi: H_{1}(X ; \mathbb{Z}) \rightarrow G$ be defined by $\varphi\left(y_{2}\right)=t$ and $\varphi\left(y_{3}\right)=1$. The following relations hold in $H_{1}^{\varphi}(\Sigma ; R)$, with $R=\mathbb{Z}\left[t^{ \pm 1}\right]$ or $\mathbb{Q}(t)$, assuming the lifts of the curves all start at the same lift of the point $\star$ :

$$
\begin{array}{lll}
\alpha_{1}=x_{1} & \beta_{1}=x_{2} & \gamma_{1}=y_{1} \\
\alpha_{2}=t^{-1} x_{2} & \beta_{2}=x_{1}-x_{3} & \gamma_{2}=x_{2} \\
\alpha_{3}=-t^{-1} x_{3}+x_{4} & \beta_{3}=x_{1}-x_{4} & \gamma_{3}=x_{3}-3 y_{3} \\
\alpha_{4}=t^{-1} y_{3}+y_{4} & \beta_{4}=y_{1}+y_{3}+y_{4} & \gamma_{4}=x_{4}+3 y_{4}
\end{array}
$$

In the cellular decomposition of $\Sigma$ given by Figure 8 , the only 2-cell has boundary $\partial \Sigma=\left[y_{1}^{-1}, x_{1}\right]\left[y_{4}^{-1}, x_{4}\right]\left[y_{2}^{-1}, x_{2}\right]\left[y_{3}^{-1}, x_{3}\right]$. This provides a single relation in $H_{1}^{\varphi}(\Sigma ; R)$ : $r=(1-t) x_{2}$. Hence by Theorem 2.3 .

- $H_{1}^{\varphi}\left(X ; \mathbb{Z}\left[t^{ \pm 1}\right]\right) \simeq \frac{\mathbb{Z}}{3 \mathbb{Z}}$ is generated by $y_{3}$ and $H_{1}^{\varphi}(X ; \mathbb{Q}(t))=0$,

- $H_{2}^{\varphi}(X ; R)=0$,

- $H_{3}^{\varphi}\left(X ; \mathbb{Z}\left[t^{ \pm 1}\right]\right) \simeq \frac{\mathbb{Z}\left[t^{ \pm 1}\right]}{\langle 1-t\rangle}$ is generated by $x_{2}$ and $H_{3}^{\varphi}(X ; \mathbb{Q}(t))=0$. 
TORSIONS AND INTERSECTION FORMS OF 4-MANIFOLDS FROM TRISECTION DIAGRAMS 21

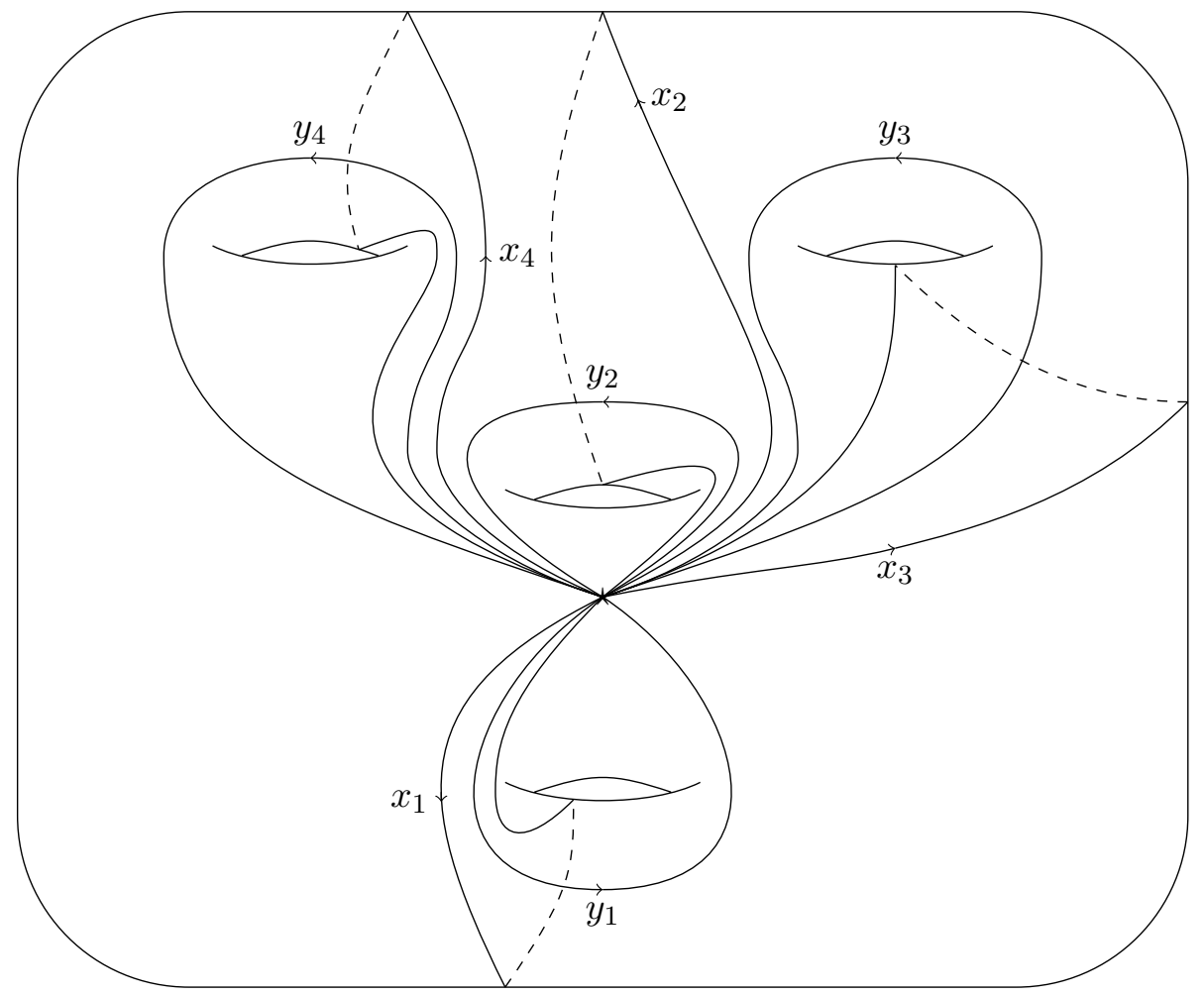

Figure 8. A basis for $\pi_{1}(\Sigma, \star)$

We now compute the torsion. Set $\Lambda=\mathbb{Z}\left[t^{ \pm 1}\right]$ and $\mathbb{F}=\mathbb{Q}(t)$. The manifold $X$ has no homology over $\mathbb{F}$. Set $u=y_{2}$. By Proposition 7.4 , the torsion $\tau^{\varphi}(X) \in \mathbb{F} / \Lambda^{*}$ is given by $\tau^{\varphi}(X)=(t-1)^{-1} \tau^{\varphi}(\widehat{X}, \star ; \widehat{h})$ where $\widehat{h}=(r, \emptyset, u)$. By Theorem [7.2, $\tau^{\varphi}(\widehat{X}, \star ; \widehat{h})$ equals the torsion $\tau(\widehat{C} ; \widehat{c}, \widehat{h})$ of the complex $\widehat{C}^{\mathbb{F}}$ :

$$
0 \rightarrow \oplus_{\nu \neq \nu^{\prime}}\left(\widehat{L}_{\nu}^{\mathbb{F}} \cap \widehat{L}_{\nu^{\prime}}^{\mathbb{F}}\right) \stackrel{\zeta}{\longrightarrow} \oplus_{\nu} \widehat{L}_{\nu}^{\mathbb{F}} \stackrel{\iota}{\longrightarrow} H_{1}^{\varphi}(\widehat{\Sigma}, \star ; \mathbb{F}) \rightarrow 0,
$$

where $\nu, \nu^{\prime}$ run over $\{\alpha, \beta, \gamma\}$ and $\widehat{c}$ is a $\Lambda$-basis over $\mathbb{F}$.

$$
\begin{aligned}
& \widehat{L}_{\alpha}^{\mathbb{F}}=\left\langle x_{2}, x_{1}, x_{3}-t x_{4}, y_{3}+t y_{4}\right\rangle \quad \widehat{L}_{\alpha}^{\mathbb{F}} \cap \widehat{L}_{\beta}^{\mathbb{F}}=\left\langle x_{2},(t-1) x_{1}+x_{3}-t x_{4}\right\rangle \\
& \widehat{L}_{\beta}^{\mathbb{F}}=\left\langle x_{2}, x_{1}-x_{3}, x_{1}-x_{4}, y_{1}+y_{3}+y_{4}\right\rangle \quad \widehat{L}_{\beta}^{\mathbb{F}} \cap \widehat{L}_{\gamma}^{\mathbb{F}}=\left\langle x_{2},-x_{3}+x_{4}+3\left(y_{1}+y_{3}+y_{4}\right)\right\rangle \\
& \widehat{L}_{\gamma}^{\mathbb{F}}=\left\langle x_{2}, y_{1}, x_{3}-3 y_{3}, x_{4}+3 y_{4}\right\rangle \quad \widehat{L}_{\gamma}^{\mathbb{F}} \cap \widehat{L}_{\alpha}^{\mathbb{F}}=\left\langle x_{2}, x_{3}-t x_{4}-3 y_{3}-3 t y_{4}\right\rangle
\end{aligned}
$$

As in the first example, fix bases $\widehat{c}, \widehat{b}$ and $\bar{b}$ to compute $\tau\left(\widehat{C}^{\mathbb{F}} ; \widehat{c}, \widehat{h}\right)$. The computation gives $\tau^{\varphi}(X)=1-t \in \mathbb{F} / \Lambda^{*}$.

\section{REFERENCES}

[Bla57] R. C. Blanchfield, Intersection theory of manifolds with operators with applications to knot theory, Annals of Mathematics. Second Series 65 (1957), 340-356. 
[FKSZ18] P. Feller, M. Klug, T. Schirmer, and D. Zemke, Calculating the homology and intersection form of a 4-manifold from a trisection diagram, arXiv: 1711.04762 (2018).

[GK16] D. Gay and R. Kirby, Trisecting 4-manifolds, Geometry \& Topology 20 (2016), no. 6, 30973132.

[KL99] Paul Kirk and Charles Livingston, Twisted Alexander invariants, Reidemeister torsion, and Casson-Gordon invariants, Topology 38 (1999), no. 3, 635-661.

[Koe17] D. Koenig, Trisections of 3-manifold bundles over $S^{1}$, arXiv: 1710.04345 (2017).

[LP72] F. Laudenbach and V. Poénaru, A note on 4-dimensional handlebodies, Bulletin de la Société Mathématique de France 100 (1972), 337-344.

[Mil66] J. Milnor, Whitehead torsion, Bulletin of the American Mathematical Society 72 (1966), $358-426$.

[Ran97] A. Ranicki, The maslov index and the Wall signature non-additivity invariant, Unpublished (1997), https://www.maths.ed.ac.uk/ v1ranick/papers/maslov.pdf

[Rei39] K. Reidemeister, Durchschnitt und Schnitt von Homotopieketten, Monatshefte für Mathematik und Physik 48 (1939), 226-239.

[Tur01] V. Turaev, Introduction to combinatorial torsions, Lectures in Mathematics ETH Zürich, Birkhäuser Verlag, Basel, 2001, Notes taken by Felix Schlenk.

[Wal69] C. T. C. Wall, Non-additivity of the signature, Inventiones Mathematicae 7 (1969), 269-274. 